\title{
Probabilistic Model Checking of Deadline Properties in the IEEE 1394 FireWire Root Contention Protocol
}

\author{
Marta Kwiatkowska ${ }^{1}$, Gethin Norman ${ }^{1}$ and Jeremy Sproston ${ }^{2}$ \\ ${ }^{1}$ School of Computer Science, University of Birmingham, Birmingham, UK \\ ${ }^{2}$ Dipartimento di Informatica, Università di Torino, Torino, Italy
}

\begin{abstract}
The interplay of real time and probability is crucial to the correctness of the IEEE 1394 FireWire root contention protocol. We present a formal verification of the protocol using probabilistic model checking. Rather than analyse the functional aspects of the protocol, by asking such questions as 'Will a leader be elected?', we focus on the protocol's performance, by asking the question 'How certain are we that a leader will be elected sufficiently quickly?' Probabilistic timed automata are used to formally model and verify the protocol against properties which require that a leader is elected before a deadline with a certain probability. We use techniques such as abstraction, reachability analysis and integer-time semantics to aid the model-checking process, and the efficacy of these techniques is compared.
\end{abstract}

Keywords: IEEE standard; FireWire; Probabilistic model checking; Timed automata

\section{Introduction}

The increasing dependence of businesses on distributed architectures and computer networking places heavy demands on the speed and reliability of data exchange, leading to the emergence of sophisticated protocols which involve both real time and randomisation, for example those used in the FireWire IEEE 1394 standard. This paper considers an application of model-checking techniques to the FireWire IEEE 1394 root contention protocol, in which the interplay of timed and probabilistic aspects is used to break the symmetry which may arise during the leader election process. Here, we are interested in establishing properties concerning the election of a leader within a certain deadline, and with a certain probability or greater.

Automatic verification techniques, including model checking, have been adapted to probabilistic timed systems in other studies [ACD91, deA98, BKH99, KNS02b]. In order to model the FireWire IEEE 1394 root contention protocol, we use the specification formalism of probabilistic timed automata [KNS02b], a variant of timed automata [AID94] extended with discrete probability distributions. The formalism is both sufficiently expressive to describe formally both the timed and probabilistic aspects of the protocol, and is amenable 
to model checking against probabilistic timed temporal logic properties. In contrast to the models based on stochastic processes [ACD91, BKH99], probabilistic timed automata exhibit non-determinism, which can be used to represent the unknown timing delays of the protocol. Furthermore, unlike the model of [deA98], probabilistic timed automata can be verified against deadline properties.

A probabilistic timed automaton comprises a finitary, probabilistic transition system equipped with a finite set of real-valued variables which increase at the same rate as real time. The presence of such clock variables means that, in a continuous-time semantic interpretation, the underlying state space of a probabilistic timed automaton is infinite. Hence, approaches for obtaining finite-state representations of such models which are faithful with respect to the validity of a class of properties are necessary. We make use of three such approaches in the context of verifying the IEEE 1394 root contention protocol against deadline properties: the first uses an algorithm to conduct a forward search through the state space of the probabilistic timed automaton, the second employs 'region equivalence' [AlD94] to partition the state space, and the third uses an integer-time semantic interpretation of the model. In each of the three cases, we use the probabilistic model-checking tool PRISM [KNP02, Pri] in the final step of establishing or refuting the relevant probabilistic deadline property.

\subsection{Related Work}

Formal verification of the IEEE 1394 root contention protocol has been performed in a number of previous studies. A probabilistic guarded command language is used in Fidge and Shankland [FiS01] to model the protocol, following which manual proof methods are used to obtain a relationship between the number of attempts to resolve root contention and the probability of successful leader election. The approach of Stoelinga and Vaandrager [StV99] is to consider a probabilistic timed model of the protocol, which is then verified manually through a process of stepwise abstraction. Such a refinement process is repeated in Simons and Stoelinga [SiS01], in which the model takes the form of a non-probabilistic timed automaton, with the real-time model checker UPPAAL [LPY97] being used to establish automatically abstraction/refinement relations. Parametric real-time model checking is performed in Hune et al. [HRS01], Collomb-Annichini and Sighireanu [CoS01] and also by Bandini et al. [BST00], in which probabilistic behaviour is modelled by fairness requirements. Our aims are different from these case studies: they do not consider deadline properties, with their emphasis concerning traditional temporal logic properties rather than probabilistic properties. In this sense, our approach has more in common with the discrete-event simulation performed on a stochastic process algebra model of the protocol in D'Argenio [DAr99].

All three verification techniques featured in this paper have precedents from the field of non-probabilistic timed automata. The first decidability results for timed automata were obtained using region equivalence [AlD94], which we also use in this paper. Forward reachability algorithms for the verification of timed automata are implemented in the tools UPPAAL [LPY97] and KronOs [DOT96, DaT98]. Furthermore, building on the theoretical basis of Henzinger et al. [HMP92] and Asarin et al. [AMP98], several case studies have been successfully verified using integer semantics [BMT99, Bey01].

\subsection{Plan of the Paper}

In Section 2, we introduce probabilistic timed automata, and show how this formalism can be used to model the root contention protocol in Section 3. Section 4 presents a number of methods to verify the protocol, and Section 5 summarises the results and offers some directions for future research.

\section{Probabilistic Timed Automata}

In this section, we introduce probabilistic timed automata [KNS02b] as our model for the IEEE 1394 root contention protocol. Probabilistic timed automata are an extension of timed automata [AlD94] (henceforth referred to as classical timed automata) with the ability to express relative likelihoods of state transitions. A classical timed automaton consists of a finitary directed control graph, the nodes of which are called locations, equipped with a finite set of real-valued variables called clocks, which are interpreted as increasing at the same rate as real time. The edges of the control graph are enabled or forced to be taken depending on whether certain constraints on the clocks are satisfied. Furthermore, a set of clocks may be reset when an edge is 
executed. Probabilistic timed automata are timed automata for which discrete probability distributions range over the edges of the control graph.

\subsection{Syntax of Probabilistic Timed Automata}

Time, Clocks and Zones. Let $\mathbb{T} \in\{\mathbb{R}, \mathbb{N}\}$ be the time domain of either the non-negative reals $\mathbb{R}$ or the naturals $\mathbb{N}$. Let $\mathscr{X}$ be a finite set of variables called clocks which take values from the time domain $\mathbb{T}$. A point $v \in \mathbb{T}^{|\mathscr{X}|}$ is referred to as a clock valuation. We use $\mathbf{0} \in \mathbb{T}^{|\mathscr{X}|}$ to denote the clock valuation which assigns 0 to all clocks in $\mathscr{X}$. Let $v \in \mathbb{T}^{|\mathscr{X}|}$ be a clock valuation, and let $t \in \mathbb{T}$ be a time duration; then the clock valuation $v \oplus t$ denotes the time increment for $v$ and $t$ (we present two alternatives for $\oplus$ in Section 2.2, one of which is standard addition + ). We use $v[X:=0]$ to denote the clock valuation obtained from the clock valuation $v \in \mathbb{T}^{|\mathscr{X}|}$ by resetting all of the clocks in $X \subseteq \mathscr{X}$ to 0 , and leaving the values of all other clocks unchanged.

Let Zones $(\mathscr{X})$ be the set of zones over $\mathscr{X}$, which are conjunctions of atomic constraints of the form $x \sim c$ and $x-y \sim c$, for clocks $x, y \in \mathscr{X}$, comparison operator $\sim \in\{<, \leqslant, \geqslant,>\}$, and naturals $c \in \mathbb{N}$. A zone $\zeta$ is diagonal-free if it does not feature a conjunct of the form $x-y \sim c$, and is closed if it does not feature a conjunct of the form $x-y \sim c$ or $x \sim c$ for $\sim \in\{\langle\rangle$,$\} . The clock valuation v$ satisfies the zone $\zeta$, written $v \triangleleft \zeta$, if and only if $\zeta$ resolves to true after replacing each clock $x \in \mathscr{X}$ with the corresponding clock value $v_{x}$ from $v$.

Probability Distributions. A discrete probability distribution over a countable set $Q$ is a function $\mu: Q \rightarrow[0,1]$ such that $\sum_{q \in Q} \mu(q)=1$. For a possibly uncountable set $Q^{\prime}$, let $\operatorname{Dist}\left(Q^{\prime}\right)$ be the set of distributions over countable subsets of $Q^{\prime}$. For any element $q \in Q$, let $\mu_{q} \in \operatorname{Dist}(Q)$ be the distribution which assigns probability 1 to $q$.

Definition 2.1. (Probabilistic Timed Automata.) A probabilistic timed automaton is a tuple PTA $=(L, \mathscr{X}, \Sigma, i n v$, prob) where:

- $L$ is a finite set of locations;

- $\Sigma$ is a finite set of events;

- the function inv $: L \rightarrow Z$ Zones $(\mathscr{X})$ is the invariant condition;

- the finite set prob $\subseteq L \times \operatorname{Zones}(\mathscr{X}) \times \Sigma \times \operatorname{Dist}\left(2^{\mathscr{X}} \times L\right)$ is the probabilistic edge relation. A probabilistic edge takes the form of a tuple $(l, g, \sigma, p) \in$ prob, where $l$ is the source location of the probabilistic edge, $g$ is its enabling condition, $\sigma$ is its event, and $p \in \operatorname{Dist}\left(2^{\mathscr{X}} \times L\right)$ is its edge distribution.

A probabilistic timed automaton is diagonal-free (closed) if all the zones used in its description are diagonalfree (closed).

A state of a probabilistic timed automaton PTA is a pair $(l, v)$ where $l \in L$ and $v \in \mathbb{T}^{|\mathscr{X}|}$ such that $v \triangleleft \operatorname{inv}(l)$. If the current state is $(l, v)$, there is a non-deterministic choice of either letting time pass while satisfying continuously the invariant condition $i n v(l)$, or making a discrete transition according to any probabilistic edge in prob with source location $l$ and whose enabling condition $g$ is satisfied by the current clock valuation $v$. If the probabilistic edge $(l, g, \sigma, p)$ is chosen, then the probability of moving to the location $l^{\prime}$ and resetting all of the clocks in the set $X$ to 0 is given by $p\left(X, l^{\prime}\right)$. The semantics of probabilistic timed automata are presented formally in the next section.

Note that we sometimes identify a designated initial location $\bar{l} \in L$, with the intuition that the behaviour of the model commences in $\bar{l}$ with all clocks set to 0 . The initial state $(\bar{l}, \mathbf{0})$, in which the value of all of the clocks is 0 , can then be used when considering reachability properties; for example, 'Is the probability of reaching a certain set of states from the initial state greater than $\lambda$ ?'

Higher-Level Modelling. To aid higher-level modelling, we can designate certain locations as being urgent; once an urgent location is entered, it must be left immediately, without time passing. The notion of urgency for locations is closely related to the concept of urgent transitions [HHW95, DaY95] (an urgent location is a location for which all outgoing discrete transitions are urgent). Urgent locations can be represented syntactically using the framework given in Definition 2.1 using an additional clock, combined with altered clock resets and invariant conditions.

It is often convenient to define systems as the parallel composition of a number of interacting subcomponents. For example, in the case of the IEEE 1394 root contention protocol, it suffices to construct 


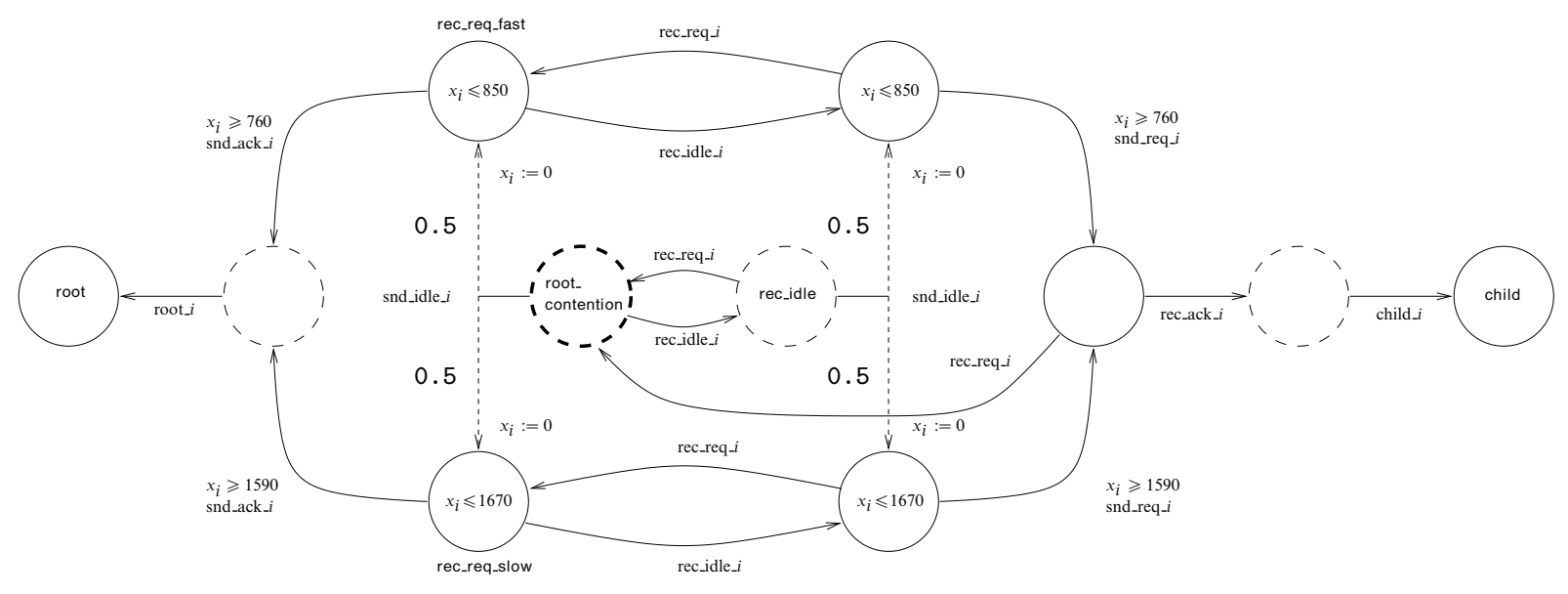

Fig. 1. The probabilistic timed automaton $\operatorname{Node}_{i}^{\mathrm{p}}$.

models for each of the two contending nodes, and for the two wires along which they communicate, given that the manner in which they interact is defined explicitly. Using ideas from the theory of (untimed) probabilistic systems [SeL95] and classical timed automata [AlD94], the parallel composition of two probabilistic timed automata, which interact by synchronising on common events, is defined in the following way. Let PTA $A_{i}=$ $\left(L_{i}, \mathscr{X}_{i}, \Sigma_{i}, i n v_{i}, \operatorname{prob}_{i}\right)$ for $i \in\{1,2\}$.

Definition 2.2. The parallel composition of two probabilistic timed automata PTA $\mathrm{A}_{1}$ and $\mathrm{PTA}_{2}$, where $\mathscr{X}_{1} \cap$ $\mathscr{X}_{2}=\emptyset$, is the probabilistic timed automaton PTA $\|$ PTA ${ }_{2}=\left(L_{1} \times L_{2}, \mathscr{X}_{1} \cup \mathscr{X}_{2}, \Sigma_{1} \cup \Sigma_{2}\right.$, inv, prob $)$ where $\operatorname{inv}\left(l, l^{\prime}\right)=\operatorname{inv}_{1}(l) \wedge \operatorname{inv}_{2}\left(l^{\prime}\right)$ for all $\left(l, l^{\prime}\right) \in L_{1} \times L_{2}$ and $\left(\left(l_{1}, l_{2}\right), g, \sigma, p\right) \in$ prob if and only if one of the following conditions holds:

- $\sigma \in \Sigma_{1} \backslash \Sigma_{2}$ and there exists $\left(l_{1}, g, \sigma, p_{1}\right) \in$ prob $_{1}$ such that $p=p_{1} \otimes \mu_{\left(\emptyset, l_{2}\right)}$;

- $\sigma \in \Sigma_{2} \backslash \Sigma_{1}$ and there exists $\left(l_{2}, g, \sigma, p_{2}\right) \in \operatorname{prob}_{2}$ such that $p=\mu_{\left(\emptyset, l_{1}\right.} \otimes p_{2}$;

- $\sigma \in \Sigma_{1} \cap \Sigma_{2}$ and there exists $\left(l_{1}, g_{1}, \sigma, p_{1}\right) \in \operatorname{prob}_{1}$ and $\left(l_{2}, g_{2}, \sigma, p_{2}\right) \in \operatorname{prob}_{2}$ such that $g=g_{1} \wedge g_{2}$ and $p=p_{1} \otimes p_{2}$

where for any $l_{1} \in L_{1}, l_{2} \in L_{2}, X_{1} \subseteq \mathscr{X}_{1}$ and $X_{2} \subseteq \mathscr{X}_{2}$, we let $p_{1} \otimes p_{2}\left(X_{1} \cup X_{2},\left(l_{1}, l_{2}\right)\right)=p_{1}\left(X_{1}, l_{1}\right) \cdot p_{2}\left(X_{2}, l_{2}\right)$.

Given the initial locations $\bar{l}_{i} \in L_{i}$ of PTA ${ }_{i}$, for $i \in\{1,2\}$, we let $\left(\bar{l}_{1}, \bar{l}_{2}\right)$ be the initial location of $\mathrm{PTA}_{1} \| \mathrm{PTA} \mathrm{A}_{2}$.

Example. Figure 1 shows the probabilistic timed automaton Node $_{i}^{\mathrm{p}}$ for a contending node of the network involved in the IEEE 1394 FireWire root contention protocol. The model Node ${ }_{i}^{\mathrm{p}}$ is a minor, probabilistic extension of the classical timed automaton node model from [SiS01]. The usual conventions for the graphical representation of classical timed automata are used (note that we omit invariant conditions or guards of the form true, and some location names, for simplicity). Figure 1 also features bifurcating edges leaving the locations root_contention (the initial location, as denoted by the bold node) and rec_idle, which correspond to probabilistic transitions. For example, the left-hand edges leaving root_contention correspond to a probabilistic choice of taking a transition to either of the target locations, rec_req_fast and rec_req_slow, each with probability 0.5 (while resetting the clock $x_{i}$ ). For simplicity, we omit the probability labels from edges corresponding to probability 1 . Urgent locations are indicated by the dashed locations.

The behaviour of the model commences in location root_contention, which models the situation in which the node has detected root contention. Because the location root_contention is urgent, Node $e_{i}^{\mathrm{p}}$ is forced to select an outgoing probabilistic edge instantly. Consider the bifurcating probabilistic edge labelled by snd_idle_i, which corresponds to the node flipping a coin in order to determine whether it should wait for a short or long time. The snd_idle_ $i$ event is sent by Node ${ }_{i}^{\mathrm{p}}$ to its communication medium, referring to a transmission of an idle signal across the node's wire to the other node. In both of the two locations rec_req_fast and rec_req_slow which may be reached after taking the probabilistic edge, the passage of time may mean that the value of the clock $x_{i}$ can reach a value enabling the left-pointing edges, in turn meaning that an acknowledgement is sent (event snd_ack $\_$), and the node then declares itself to be leader (the event root_ $i$ 
which labels the subsequent left-pointing edge to the location root). In contrast, if the node receives an idle signal from the other contending node (event rec_idle_i) before sending an acknowledgement, it is forced to move to the right via an edge labelled with the event rec_idle $i$. In this case, after a certain amount of time elapses, Node ${ }_{i}^{\mathrm{p}}$ can send a request to the other node to be its parent by sending the event snd_req_i to its wire. If the node then subsequently detects a parent request from the other node (event rec_req_i), it returns to the location root_contention, and restarts the root contention process. If, on the other hand, the node detects an acknowledgement from the other node (event rec_ack_i), it proceeds to declare itself as the child by sending a child $\_i$ event.

\subsection{Semantics of Probabilistic Timed Automata}

\subsubsection{Probabilistic Systems}

The semantics of probabilistic timed automata is defined in terms of transition systems exhibiting both non-deterministic and probabilistic choice. We call such models probabilistic systems, noting that they are essentially equivalent to Markov decision processes [Der70], the simple probabilistic automata of Segala and Lynch [SeL95], and the probabilistic-nondeterministic systems of Bianco and de Alfaro [Bid95].

Definition 2.3. (Probabilistic Systems.) A probabilistic system $\mathrm{PS}=(S$, Act, Steps $)$ consists of a set $S$ of states, a set Act of actions, and a probabilistic transition relation Steps $\subseteq S \times A c t \times \operatorname{Dist}(S)$.

A probabilistic transition $s \stackrel{a, \mu}{\longrightarrow} s^{\prime}$ is made from a state $s \in S$ by first non-deterministically selecting an actiondistribution pair $(a, \mu)$ such that $(s, a, \mu) \in$ Steps, and second by making a probabilistic choice of target state $s^{\prime}$ according to $\mu$, such that $\mu\left(s^{\prime}\right)>0$. In the sequel, probabilistic systems may be infinite-state.

We now give the semantics of probabilistic timed automata defined in terms of probabilistic systems. As with classical timed automata, transitions consist of two types: time transitions, which correspond to the passage of time while the current location remains constant, and discrete transitions, which correspond to a probabilistic edge being taken. The definition is parameterised both by a time domain $\mathbb{T}$ and a time increment $\oplus$.

Definition 2.4. (Semantics of Probabilistic Timed Automata.) The semantics of a probabilistic timed automaton PTA $=(L, \mathscr{X}, \Sigma$, inv, prob $)$ with respect to the time domain $\mathbb{T}$ and the time increment $\oplus$ is the probabilistic system $\llbracket \mathrm{PTA} \rrbracket_{\mathbb{T}}^{\oplus}=(S$, Act, Steps $)$ defined by the following.

States. Let $S \subseteq L \times \mathbb{T}^{|\mathscr{X}|}$ such that $(l, v) \in S$ if and only if $v \triangleleft i n v(l)$.

Actions. Let $A c t=\mathbb{T} \cup \Sigma$.

Probabilistic Transitions. Let Steps be the least set of probabilistic transitions defined as follows. For each state $(l, v) \in S:$

- Time transitions. For each duration $t \in \mathbb{T}$, let $((l, v), t, \mu) \in$ Steps if and only if $(1) v \oplus t^{\prime} \triangleleft i n v(l)$ for each $0 \leqslant t^{\prime} \leqslant t$, and $(2) \mu(l, v \oplus t)=1$.

- Discrete transitions. For each probabilistic edge $(l, g, \sigma, p) \in \operatorname{prob}$, let $((l, v), \sigma, \mu) \in$ Steps if and only if $v \triangleleft g$, for each state $\left(l^{\prime}, v^{\prime}\right) \in S$ :

$$
\mu\left(l^{\prime}, v^{\prime}\right)=\sum_{X \subseteq \mathscr{X} \& v^{\prime}=v[X:=0]} p\left(X, l^{\prime}\right)
$$

and for each $\left(X, l^{\prime}\right) \in 2^{\mathscr{X}} \times L$ such that $p\left(X, l^{\prime}\right)>0$, we have $v[X:=0] \triangleleft \operatorname{inv}\left(l^{\prime}\right)$.

The summation in the definition of discrete transitions is required for the cases in which multiple clock resets result in the same target state $\left(l^{\prime}, v^{\prime}\right)$. Furthermore, the final clause is required to preclude the pathological situation in which an invariant of a location is not satisfied directly after a probabilistic transition.

The semantics falls into two classes, depending on whether the underlying model of time is the positive reals or the naturals. In the sequel, we always let $\oplus$ equal + if $\mathbb{T}=\mathbb{R}$. We refer to $\llbracket \mathrm{PTA} \rrbracket_{\mathbb{R}}^{+}$as the continuous semantics of the probabilistic timed automaton PTA. In contrast, if $\mathbb{T}=\mathbb{N}$, we always let $\oplus$ equal $\oplus_{\mathbb{N}}$, which is defined as follows. Let PTA be a probabilistic automaton; for any $x \in \mathscr{X}$, let $\mathbf{k}_{x}$ denote the greatest constant the clock $x$ is compared to in the zones of PTA. Then, for any clock valuation $v \in \mathbb{N}^{|\mathscr{X}|}$ and time duration 
$t \in \mathbb{N}$, let $v \oplus_{\mathbb{N}} t$ be the clock valuation of $\mathscr{X}$ which assigns the value $\min \left\{v_{x}+t, \mathbf{k}_{x}+1\right\}$ to all clocks $x \in \mathscr{X}$ (although the operator $\oplus_{\mathbb{N}}$ is dependent on PTA, we elide a sub- or superscript indicating this for clarity). Then we refer to $\llbracket P T A \rrbracket_{\mathbb{N}}^{\oplus_{N}}$ as the integer semantics of PTA. The definition of integer semantics for probabilistic timed automata is a generalisation of the analogous definition for the classical model in Beyer [Bey01], which in turn follows the methodology of other studies [HMP92, AMP98, BMT99]. As we always use the same definition of time increment for a particular choice of time domain, we omit the + and $\oplus_{\mathbb{N}}$ superscripts from the notation for the continuous and discrete semantics for simplicity.

Note that the fact that the integer semantics of a probabilistic timed automaton is finite, and the continuous semantics of probabilistic timed automaton is generally infinite, can be derived from the definitions. In both cases, all states have at least one available transition (namely, the time transition $\left(s, 0, \mu_{s}\right)$ ).

It is not difficult to check that the semantics of the parallel composition of two probabilistic timed automata corresponds to the semantics of the parallel composition of their individual semantic probabilistic systems. Formally, we overload the parallel composition operator $\|$ such that $\mathrm{PS}_{1} \| \mathrm{PS}_{2}$ denotes the probabilistic system obtained from the parallel composition of the probabilistic systems $\mathrm{PS}_{1}$ and $\mathrm{PS}_{2}$ in the standard manner [SeL95]. Two probabilistic systems $\left.\mathrm{PS}_{1}=\left(S_{1}, \text { Act, Steps }\right)_{1}\right)$ and $\left.\mathrm{PS}_{2}=\left(S_{2}, \text { Act, Steps }\right)_{2}\right)$ are isomorphic if there exists a bijection $f: S_{1} \rightarrow S_{2}$ such that $\left(s_{1}, a, \mu\right) \in$ Steps $_{1}$ if and only if $\left(f\left(s_{1}\right), a, f(\mu)\right) \in$ Steps $_{2}$, where $f(\mu) \in \operatorname{Dist}\left(S_{2}\right)$ is the distribution defined by $f(\mu)\left(s_{2}\right)=\mu\left(f^{-1}\left(s_{2}\right)\right)$ for each $s_{2} \in S_{2}$. For the probabilistic timed

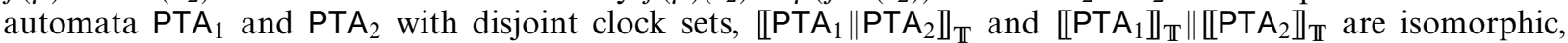
both for the continuous and integer semantics.

\subsubsection{Behaviour of Probabilistic Timed Automata}

The behaviour of a probabilistic timed automaton is described in terms of the behaviour of its (continuous or integer) semantic probabilistic system. We consider two ways in which a probabilistic system's computation may be represented, followed by definitions of probability measures of interest.

Paths. A path represents a particular resolution of both non-determinism and probability of a probabilistic system. Formally, a path of a probabilistic system $\mathrm{PS}=(S, A c t$, Steps $)$ is a non-empty finite or infinite sequence of transitions $\omega=s_{0} \stackrel{a_{0}, \mu_{0}}{\longrightarrow} s_{1} \stackrel{a_{1}, \mu_{1}}{\longrightarrow} \cdots$. We use $P a t h_{f i n}^{\mathrm{PS}}$ to denote the set of finite paths of PS, and Path ful the set of infinite paths of PS.

For a path $\omega$ and $i \in \mathbb{N}$, we denote by $\omega(i)$ the $(i+1)$ th state of $\omega$; if $\omega$ is finite, we denote by last $(\omega)$ the last state of $\omega$. We abuse notation by letting a state $s \in S$ to denote a path consisting of no transitions, and by using $\omega \stackrel{a, \mu}{\longrightarrow} S$ to refer to a path comprising the sequence of transitions of $\omega$ followed by the transition last $(\omega) \stackrel{a, \mu}{\longrightarrow} s$.

Adversaries. An adversary represents a particular resolution of non-determinism only. Formally, an adversary of a probabilistic system PS is a function $A$ mapping every finite path $\omega \in \operatorname{Path}_{\text {fin }}^{\text {PS }}$ to a pair $(a, \mu) \in A c t \times \operatorname{Dist}(S)$ such that $(\operatorname{last}(\omega), a, \mu) \in$ Steps [Var85]. Let $A d v_{\mathrm{PS}}$ be the set of adversaries of PS.

Probability measures. For any adversary $A \in A d v_{\mathrm{PS}}$, let $\mathrm{Path}_{\text {fin }}^{A}$ and $\mathrm{Path}_{\text {ful }}^{A}$ denote the set of finite and infinite paths associated with $A$ (more precisely, the paths resulting from the choices of action-distribution pairs of $A$ ). Then we define the probability measure $\operatorname{Prob}^{A}$ over Path $_{f u l}^{A}$ in the following, standard way [KSK76]. First we define the function A : Path ${ }_{\text {fin }}^{A} \times \operatorname{Path}_{\text {fin }}^{A} \rightarrow[0,1]$, such that:

$$
\mathrm{A}\left(\omega, \omega^{\prime}\right)=\left\{\begin{array}{cl}
\mu(s) & \text { if } A(\omega)=(\operatorname{last}(\omega), a, \mu) \text { for some } a \in A c t, \text { and } \omega^{\prime}=\omega \stackrel{a, \mu}{\longrightarrow} s \\
0 & \text { otherwise }
\end{array}\right.
$$

Intuitively, $A\left(\omega, \omega^{\prime}\right)$ refers to the probability of obtaining the finite path $\omega^{\prime}$ when extending the finite path $\omega$ with one transition under the control of the adversary $A$. Next, the function $\operatorname{Prob}_{\text {fin }}^{A}:$ Path fin $^{A} \rightarrow[0,1]$ is defined inductively along the length of finite paths of $A$ as follows:

- let $\operatorname{Prob}_{f i n}^{A}(s)=1$ for all states $s \in S$ (paths of length 0 );

- if $(\omega \stackrel{a, \mu}{\longrightarrow} s) \in \operatorname{Path}_{f i n}^{A}$, then $\operatorname{Prob}_{f i n}^{A}(\omega \stackrel{a, \mu}{\longrightarrow} s)=\operatorname{Prob}_{f i n}^{A}(\omega) \cdot \mathrm{A}(\omega, \omega \stackrel{a, \mu}{\longrightarrow} s)$

For a finite path $\omega \in \operatorname{Path}_{\text {fin }}^{A}$, let Cone $(\omega)$ be the cone generated by $\omega$, defined as the set Cone $(\omega)=\left\{\omega^{\prime} \in\right.$ $\operatorname{Path}_{\text {ful }}^{A} \mid \omega$ is a prefix of $\left.\omega^{\prime}\right\}$ of infinite paths. We then define $\operatorname{Prob}^{A}$ by $\operatorname{Prob}^{A}(\operatorname{Cone}(\omega))=\operatorname{Prob}_{\text {fin }}^{A}(\omega)$, which can then be uniquely extended to a probability measure on the sigma-algebra generated by the cones of $A$. 
Reachability probabilities. The maximal (minimal) reachability probability is the maximum (minimum) probability with which a given set of states of a probabilistic system can be reached from a particular state. Formally, for the probabilistic system PS $=(S, A c t$, Steps $)$, state $s \in S$, set $F \subseteq S$ of target states, and adversary $A \in A d v$ PS, the probability of reaching $F$ from $S$ under adversary $A$ is given by:

$$
\operatorname{ProbReach}^{A}(s, F) \stackrel{\text { def }}{=} \operatorname{Prob}^{A}\left\{\omega \in \operatorname{Path}_{\text {ful }}^{A} \mid \omega(0)=s \& \exists i \in \mathbb{N} . \omega(i) \in F\right\}
$$

Then the maximal and minimal reachability probabilities $\operatorname{MaxProbReach}_{\mathrm{PS}}(\mathrm{S}, F)$ and $\operatorname{MinProbReach}_{\mathrm{PS}}(s, F)$ are defined as:

$$
\operatorname{MaxProbReach}_{\mathrm{PS}}(\mathrm{s}, F) \stackrel{\text { def }}{=} \sup _{A \in A d v \mathrm{PS}} \operatorname{ProbReach}^{A}(s, F), \quad \operatorname{MinProbReach}_{\mathrm{PS}}(s, F) \stackrel{\text { def }}{=} \inf _{A \in \operatorname{Ad}_{\mathrm{PS}}} \operatorname{ProbReach}^{A}(s, F)
$$

The following theorem is key to establishing the correctness of the integer semantics with regard to the probability of reaching a certain set of target locations of a closed, diagonal-free probabilistic timed automaton. The theorem states that both the maximal and minimal probabilities of reaching a target location are equal in the continuous and integer semantics for this class of model, and is a probabilistic extension of a similar result established in Beyer [Bey01]. Let $L^{\prime} \subseteq L$ be a set of target locations of a probabilistic timed automaton PTA, and let the set of all states corresponding to locations in $L^{\prime}$ be denoted by $F_{\mathbb{T}}^{L^{\prime}}=\left\{(l, v) \mid l \in L^{\prime}, v \in\right.$ $\left.\mathbb{T}^{|X|} \& v \triangleleft \operatorname{inv}(l)\right\}$.

Theorem 2.5. For every closed, diagonal-free probabilistic timed automata PTA $=(L, \mathscr{X}, \Sigma$, inv, prob $)$, initial location $\bar{l} \in L$, and target set $L^{\prime} \subseteq L$ of locations:

$$
\begin{aligned}
\operatorname{MaxProbReach}_{\llbracket \mathrm{PTA}}\left((\bar{l}, \mathbf{0}), F_{\mathbb{R}}^{L^{\prime}}\right) & =\text { MaxProbReach }_{\llbracket \mathrm{PTA} \rrbracket_{\mathbb{N}}}\left((\bar{l}, \mathbf{0}), F_{\mathbb{N}}^{L^{\prime}}\right) \\
\text { MinProbReach }_{\llbracket \mathrm{PTA}}\left((\bar{l}, \mathbf{0}), F_{\mathbb{R}}^{L^{\prime}}\right) & =\text { MinProbReach }_{\llbracket \mathrm{PTA}}\left((\bar{l}, \mathbf{0}), F_{\mathbb{N}}^{L^{\prime}}\right)
\end{aligned}
$$

Proof. See Appendix A.

\subsection{Time Progress in Probabilistic Timed Automata}

As with the case of classical timed automata [A1D94], some paths of a probabilistic timed automaton may correspond to pathological situations in which time does not progress beyond some bound; therefore, such computations will not be exhibited by any real-life system. The solution for classical timed automata is to design model-checking methods which disregard such Zeno paths. Following precedents from the probabilistic real-time systems literature [deA97, Seg95], the lifting of the concept of time progress from classical to probabilistic timed automata involves characterising adversaries, rather than paths, which exhibit 'non-Zenoness', and consequently disregarding all other adversaries during model checking [KNS02b].

Definition 2.6. (Non-Zenoness.) Let PTA be a probabilistic timed automaton with time domain $\mathbb{T}$, and let $\omega=\left(l_{0}, v_{0}\right) \stackrel{a_{0}, \mu_{0}}{\longrightarrow}\left(l_{1}, v_{1}\right) \stackrel{a_{1}, \mu_{1}}{\longrightarrow} \cdots$ be a path of $\llbracket \mathrm{PTA} \rrbracket_{\mathbb{T}}$. The elapsed time along $\omega$ at step $i \in \mathbb{N}$ is the sum of the durations of the time transitions of $\omega$ taken before the $i$ th transition, and is formally defined as:

$$
\text { time }(\omega, i)=\sum\left\{a_{j} \mid 0 \leqslant j<i \text { and } a_{j} \in \mathbb{T}\right\}
$$

A path $\omega$ of $\left[\mathrm{PTA} \rrbracket_{\mathbb{T}}\right.$ is non-Zeno if and only if, for all $t \in \mathbb{T}$, there exists $i \in \mathbb{N}$ such that time $(\omega, i)>t$. An adversary $A$ of $\llbracket \mathrm{PTA} \rrbracket_{\mathbb{T}}$ is non-Zeno if and only if $\operatorname{Prob}^{A}\left\{\omega \in \operatorname{Path}_{\text {ful }}^{A} \mid \omega\right.$ is non-Zeno $\}=1$.

For convenience, we also refer to paths and adversaries which are not non-Zeno as Zeno.

\section{Modelling the Root Contention Protocol}

\subsection{The Probabilistic Timed Automaton Models}

We now explain how the IEEE 1394 root contention protocol can be specified and verified formally within the framework of probabilistic timed automata. The classical timed automaton models of [SiS01] are taken as the basis for the probabilistic models introduced here. As we have seen in Section 2.1, the model Node ${ }_{i}^{\mathrm{p}}, i \in\{1,2\}$, of the $i$ th node of the protocol can be extended with probability in a straightforward manner; simply let the two 


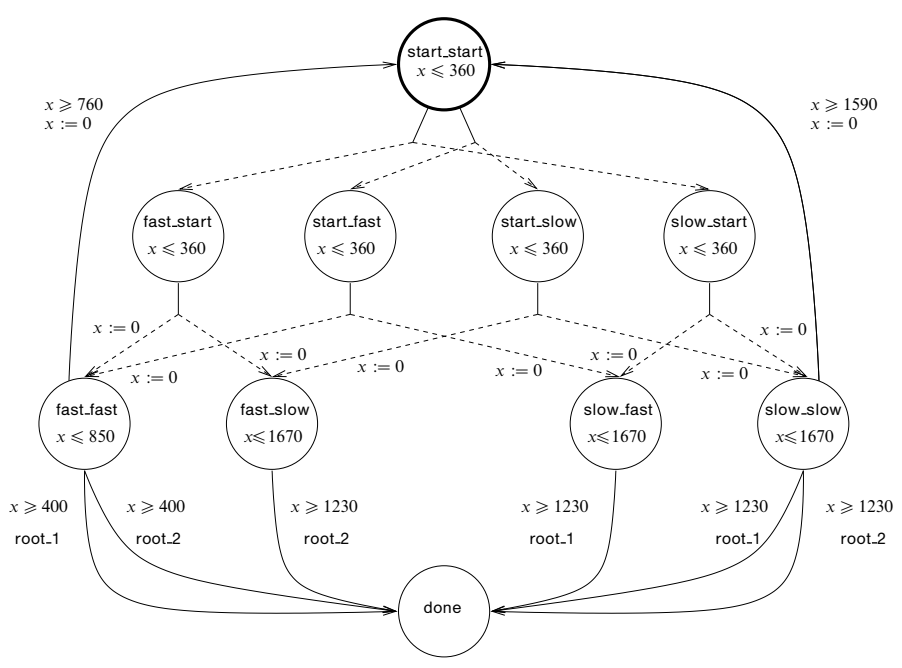

Fig. 2. The probabilistic timed automaton $I_{1}^{\mathrm{p}}$.

edges corresponding to the outcome of a coin flip each be assigned probability 0.5 by a distribution. All other edges in the node model are taken with probability 1 . The communication medium between the nodes, which assumes that signals are driven continuously across wires which comprise two-place buffers, is then represented by the models Wire ${ }_{i}$, for $i \in\{1,2\}$. The classical timed automaton models for the wires in [SiS01] are adopted directly (the interested reader is invited to refer to [SiS01]), with the interpretation that all transitions of the wire models are made with probability 1 . The parallel composition $\operatorname{Impl}^{\mathrm{p}}=$ Node $_{1}^{\mathrm{p}} \|$ Wire $_{1} \|$ Wire $_{2} \|$ Node $_{2}^{\mathrm{p}}$ of the resulting probabilistic timed automata is then defined using Definition 2.2.

As will be explained in Section 4, during initial verification experiments, it became clear that analysis of a model as complex as the composed probabilistic timed automaton is not feasible given our current implementation of the forward reachability algorithm of [KNS02b]. Therefore, we also study the smaller, more abstract, probabilistic timed automaton $I_{1}^{p}$ of the root contention protocol. The model for $I_{1}^{p}$ is shown in Fig. 2, and is a probabilistic extension of the classical timed automaton $I_{1}$ used in the stepwise refinement process of [SiS01]. The constants used in the enabling and invariant conditions are derived from those given in the IEEE 1394a standard when the communication delay between the nodes is taken to be $360 \mathrm{~ns}$. Each instance of bifurcating edges corresponds to a coin being flipped; for example, in the location start_start, there is a non-deterministic choice between node 1 (re)starting the root contention protocol and flipping its coin, leading with probability 0.5 to each of slow_start and fast_start (we omit all probability labels from the diagram for simplicity), and node 2 restarting the protocol and flipping its coin. The location done represents the election of a leader. Although it is possible for a leader to be elected regardless of the outcome of the probabilistic choices, as there are edges from each of the locations fast_fast, fast_slow, slow_fast and slow_slow to the location done, note that it is also possible to return to start_start, corresponding to protocol restart, from the locations fast_fast and slow_slow, in which the nodes' coin flips are identical. Note that the timing constant for the enabling condition of the edge from fast_fast to done is obtained from $760 \mathrm{~ns}$, the minimal waiting time if the 'fast' side of the coin is obtained, minus $360 \mathrm{~ns}$, the wire propagation delay; similarly, the enabling conditions of the other edges to done are obtained from $1590 \mathrm{~ns}$, the minimal waiting time if the 'slow' side of the coin is obtained, minus $360 \mathrm{~ns}$.

The timing constraints used in $\mathrm{I}_{1}^{\mathrm{p}}$ correspond to those specified in the updated standard IEEE 1394a. In the figure, the maximum transmission delay equals $360 \mathrm{~ns}$, which represents the assumption that the contending nodes are separated by a distance close to the maximum required for the correctness of the protocol (from the analysis of [SiS01]). However, it is straightforward to change this value and rerun the experiments, if required; we also consider a delay of $30 \mathrm{~ns}$, which corresponds more closely to the maximum separation of nodes specified in the IEEE 1394a standard. This maximum separation results in a maximum transmission delay of $22.725 \mathrm{~ns}$, and hence the choice of $30 \mathrm{~ns}$ is an overapproximation of the delay, which results in upper bounds on the clock $x$ being higher in our model (for example, we have invariant conditions of the form $x \leqslant 30$ rather than $x \leqslant 22.725$ ). Dually, lower bounds are lower in our model (for example, we have enabling conditions of the form $x \geqslant 1560$ rather than $x \geqslant 1567.275)$ than in a faithful representation of the standard. 
The choice of a transmission delay of $30 \mathrm{~ns}$ is made for efficiency reasons, as it allows us to use a time granularity of $10 \mathrm{~ns}$ when we consider probabilistic model checking based on the region graph and on integer semantics in Section 4. Finally, to measure the time elapsed since the start of system execution, we augment the probabilistic timed automaton $\mathrm{I}_{1}^{\mathrm{P}}$ with an additional clock $y$, which can be referred to in the formalisation of our deadline property.

The model $I_{1}^{\mathrm{p}}$ represents an abstraction of the root contention protocol, in the sense that it may exhibit a superset of adversaries of a more refined protocol model, such as Impl ${ }^{\mathrm{p}}$. In addition, the raising of upper bounds and the decreasing of lower bounds on clocks described in the previous paragraph is another source of abstraction of this form; for more information we refer the reader elsewhere [AIK95, KNS02a]. This means that the most unfavourable adversary with respect to the satisfaction of a property of the protocol must also be exhibited by $\mathrm{I}_{1}^{\mathrm{p}}$. Hence, as the property of interest concerns whether a leader will be elected with a certain probability or greater, for all adversaries, the minimal probability of property satisfaction that is computed for $I_{1}^{p}$ will form a lower bound on the probability in the protocol model Impl ${ }^{p}$. However, as will be seen in Section 4, the probabilities computed for the verification of the abstract model $I_{1}^{p}$ and the full model $\operatorname{Impl}{ }^{p}$ for a number of deadlines agree, suggesting (but not confirming) that $I_{1}^{p}$ does not abstract from information of $\operatorname{Impl}^{\mathrm{P}}$ relevant to the probabilistic deadline property.

\subsection{Establishing the Abstraction}

We now describe the method which is used to verify that $I_{1}^{p}$ is indeed a probabilistic, timed abstraction of the probabilistic timed automaton $\operatorname{Impl}^{\mathrm{P}}$ using 'trace refinement' or 'language containment' verification on non-probabilistic structures. This class of verification method establishes that a non-probabilistic transition system $\mathrm{TS}_{1}$ is an abstraction of another transition system $\mathrm{TS}_{2}$ (dually, that $\mathrm{TS}_{2}$ refines $\mathrm{TS}_{1}$ ) if every linear sequence of actions of $\mathrm{TS}_{2}$ is also exhibited by $\mathrm{TS}_{1}$. This technique is used to prove abstraction relations between the classical timed automata modelling the root contention protocol in [SiS01]. We now sketch how trace refinement verification on non-probabilistic, classical timed automata versions $\mathrm{TA}_{\mathrm{Impl}^{\mathrm{p}}}$ and $\mathrm{TA}_{\mathrm{I}_{1}^{\mathrm{p}}}$ of $\operatorname{Impl}^{\mathrm{p}}$ and $I_{1}^{p}$ respectively (which broadly correspond to the classical timed automata models of [SiS01]) can be used to infer probabilistic refinement between $\operatorname{Impl}{ }^{\mathrm{p}}$ and $\mathrm{I}_{1}^{\mathrm{p}}$. This is achieved by using traces of the non-probabilistic models to reconstruct paths of the probabilistic models. A more formal description of a similar procedure is given in [KNSO2a].

The method is crucially reliant on how much information from the probabilistic timed automata is encoded in the actions of the classical timed automata $\mathrm{TA}_{\mathrm{Imp} 1^{\mathrm{p}}}$ and $\mathrm{TA}_{\mathrm{I}_{1}^{\mathrm{p}}}$ (the location set and invariant conditions of the classical model equal those of the probabilistic one). An extreme case is that in which each transition $s \stackrel{a, \mu}{\longrightarrow} s^{\prime}$ of the (continuous semantics) probabilistic system $\llbracket \operatorname{Impl} \mathbb{P}_{\mathbb{R}}$ is encoded by a transition $s \stackrel{\left\langle s, a, \mu, s^{\prime}\right\rangle}{\longrightarrow} s^{\prime}$ of the (continuous semantics) transition system $\llbracket T A_{I m p 1 p} \rrbracket_{\mathbb{R}}$, thus inducing a natural one-to-one relationship between the sequence of actions (trace) $\left\langle\left\langle s_{0}, a_{0}, \mu_{0}, s_{1}\right\rangle\right\rangle\left\langle\left\langle s_{1}, a_{1}, \mu_{1}, s_{2}\right\rangle\right\rangle \cdots$ of the transition system and the path $s_{0} \stackrel{a_{0}, \mu_{0}}{\longrightarrow} s_{1} \stackrel{a_{1}, \mu_{1}}{\longrightarrow} s_{2} \cdots$ of the probabilistic system. We then perform a similar construction for $\llbracket I_{1}^{\mathrm{P}} \rrbracket_{\mathbb{R}}$ and $\left[\mathrm{TA}_{\mathrm{I}_{1}^{\mathrm{p}}} \rrbracket_{\mathbb{R}}\right.$ which differs from the one given above in the sense that the labels include information about the states of $\llbracket \operatorname{Impl} 1^{\mathrm{p}} \rrbracket_{\mathbb{R}}$ and $\left[T A_{\operatorname{Impl}} \rrbracket_{\mathbb{R}}\right.$. This is necessary to establish a meaningful notion of trace refinement in our context; otherwise we would be unable to match any action of $\llbracket T A_{I m p 1^{p}} \rrbracket_{\mathbb{R}}$ with any action of $\llbracket T A_{I_{1}^{p}} \rrbracket_{\mathbb{R}}$, and therefore we would also be unable to match traces of $\llbracket T A_{I m p 1^{p}} \rrbracket_{\mathbb{R}}$ and $\llbracket T A_{I_{1}^{p}} \rrbracket_{\mathbb{R}}$. Technically, this is achieved using a step refinement function $f$ [StV99, SiS01], which associates each state of $\llbracket T A_{I_{1}^{p}} \rrbracket_{\mathbb{R}}$ with a state of $\left[T A_{I m p 1^{p}} \rrbracket_{\mathbb{R}}\right.$, and which is defined manually using knowledge concerning the way in which $I_{1}^{p}$ is an abstraction of $\operatorname{Impl}{ }^{p}$. For example, the states with the location component start_start of $I_{1}^{p}$ could be expected to correspond to the states of $\operatorname{Impl}^{\mathrm{p}}$ with the location components root_contention and rec_idle; therefore, these states can be associated using a step refinement function.

However, as we describe systems at the level of probabilistic and classical timed automata, we cannot implement such action-renaming strategies at the level of their (infinite-state) semantic transition systems. Therefore, we choose an encoding which involves the renaming of the events labelling the probabilistic edges of the probabilistic timed automata models. For example, we replace the probabilistic, bifurcating edge (root_contention, true, snd_idle_i, $p$ ) of Node ${ }_{i}^{\mathrm{p}}$ with two edges, one labelled with the event 《 root_contention, snd_idle_ $i, p$, rec_req_fast $\rangle\rangle$, the other with $\langle\langle$ root_contention, snd_idle_ $i, p$, rec_req_slow $\rangle\rangle$. These events record the actual distribution used to make the transition $(p)$ and its outcome (rec_req_fast or rec_req_slow). This 
process is also repeated in the abstract model $\mathrm{I}_{1}^{\mathrm{p}}$, given that each location of $\mathrm{I}_{1}^{\mathrm{p}}$ is associated with certain locations of $\operatorname{Impl}{ }^{\mathrm{p}}$ via a step refinement function. That this encoding of events suffices follows from the fact that the model $\mathrm{TA}_{\mathrm{Imp}^{\mathrm{p}}}$ has the following property: given a source state and an action, the target state is uniquely or probabilistically determined, implying that, for a given initial state of the continuous semantics $\llbracket T A_{I m p 1^{p}} \rrbracket_{\mathbb{R}}$ and a finite sequence of actions of $\llbracket T A_{I m p 1^{p}} \rrbracket_{\mathbb{R}}$, the state reached after executing actions in the sequence is unique. This property induces a one-to-one relationship between traces of $\llbracket T A_{\operatorname{Imp} 1^{\mathrm{p}}} \rrbracket_{\mathbb{R}}$ and paths of $\llbracket \operatorname{Impl} 1^{\mathrm{p}} \rrbracket_{\mathbb{R}}$, as required.

Hence, if $\llbracket T A_{I_{1}^{p}} \rrbracket_{\mathbb{R}}$ is proved to be an abstraction of $\llbracket T A_{I m p 1^{p}} \rrbracket_{\mathbb{R}}$ via trace refinement, then we know that every path of $\llbracket I_{1}^{\mathrm{P}} \rrbracket_{\mathbb{R}}$ can be matched, modulo the step refinement function $f$, with a path of $\llbracket \operatorname{Impl} \mathbb{1}^{\mathrm{P}} \rrbracket_{\mathbb{R}}$. Now note that, in the construction of $\left[T A_{I_{1}^{p}} \rrbracket_{\mathbb{R}}\right.$ and $\llbracket T A_{I m p 1 p} \rrbracket_{\mathbb{R}}$, we replaced probabilistic branching with non-deterministic branching; more precisely, the alternatives available through probabilistic choice in any given state of the probabilistic system are available through non-deterministic choice in the corresponding state of the transition system. This permits us to reconstruct probabilistic branching in the following way: if, in a state $s$ of the transition system, there are the actions $\left\langle\left\langle s \stackrel{a, \mu}{\longrightarrow} s^{\prime}\right\rangle\right\rangle$ and $\left\langle\left\langle s \stackrel{a, \mu}{\longrightarrow} s^{\prime \prime}\right\rangle\right\rangle$ available, then we know that we can reconstruct the transitions $s \stackrel{a, \mu}{\longrightarrow} s^{\prime}$ and $s \stackrel{a, \mu}{\longrightarrow} s^{\prime \prime}$ of the probabilistic system, and that the probability of the first transition is $\mu\left(s^{\prime}\right)$, while the probability of the second transition is $\mu\left(s^{\prime \prime}\right)$. This permits us to construct adversaries of $\llbracket I m p I^{p} \rrbracket_{\mathbb{R}}$ and $\llbracket I_{1}^{p} \rrbracket_{\mathbb{R}}$ from sets of traces of $\llbracket T A_{I m p 1^{p}} \rrbracket_{\mathbb{R}}$ and $\llbracket T A_{I_{1}^{p}} \rrbracket_{\mathbb{R}}$, along with their associated probability measures. Then, again modulo the step refinement function $f$, we can identify adversaries of $\llbracket \mathrm{Impl}^{\mathrm{p}} \rrbracket_{\mathbb{R}}$ and $\llbracket \mathrm{I}_{1}^{\mathrm{p}} \rrbracket_{\mathbb{R}}$ with one another. Indeed, if $\llbracket T A_{I_{1}^{\mathrm{p}}} \rrbracket_{\mathbb{R}}$ is an abstraction of $\llbracket T A_{I_{m p 1^{p}} \rrbracket_{\mathbb{R}}}$, every adversary of $\llbracket I m p I^{\mathrm{P}} \rrbracket_{\mathbb{R}}$ can be matched via $f$ with an adversary of $\llbracket I_{1}^{\mathrm{p}} \rrbracket_{\mathbb{R}}$ (for those familiar with Segala's theory of probabilistic systems, such adversaries will have the same trace distributions [Seg95]). Such matching allows us to reason about properties referring to the probability of observing sequences of actions, including our deadline property. More precisely, for every adversary of $\llbracket \operatorname{Impl}^{\mathrm{p}} \rrbracket_{\mathbb{R}}$, there exists an adversary of $\llbracket \mathrm{I}_{1}^{\mathrm{p}} \rrbracket_{\mathbb{R}}$ for which the probability of a root_i event occurring within deadline time units is the same. As the converse does not necessarily hold, we can conclude that the minimal and maximal probabilities of electing a leader within the deadline obtained by analysis of $I_{1}^{p}$ form lower and upper bounds, respectively, on the probabilities of electing a leader within the deadline for $\operatorname{Impl}{ }^{\mathrm{P}}$.

After constructing the classical timed automata $T A_{I_{m p 1}}^{p}$ and $T A_{I_{1}^{p}}$ from Impl ${ }^{p}$ and $I_{1}^{p}$, we then used the methodology of [SiS01] to test that $\mathrm{TA}_{\mathrm{Imp}^{\mathrm{p}}}$ trace refines $\mathrm{TA}_{\mathrm{I}_{1}^{\mathrm{p}}}$. This result was established by automating the refinement proof using the classical timed automaton model-checking tool UPPAAL. Hence, we conclude that every adversary of $\mathrm{Impl}^{\mathrm{p}}$ can be matched via $f$ with an adversary of $\mathrm{I}_{1}^{\mathrm{p}}$, and that the minimum and maximum probabilities of satisfying the deadline property in $I_{1}^{p}$ form bounds on the corresponding probabilities obtained from Implp.

\section{Verification and Analysis}

Model-checking strategies for classical and probabilistic timed automata are crucially reliant on assumptions concerning the underlying model of time. Traditionally, timed automata are presented as a dense-time model (for example, with $\mathbb{T}=\mathbb{R}$ ), in which case the set of possible values that clocks take is infinite, and therefore the semantic probabilistic system is infinite-state. However, for a rich class of properties, including the reachability properties that we consider in this paper, a finite-state transition system to be model checked can be derived. A number of methods for obtaining such a finite-state system from a timed automaton operating in continuous time exist: one concerns partitioning the state space according to the well-established region equivalence [AlD94]; another concerns the verification of timed reachability properties by forward exploration through the state space by iterating successively transition successor relations from state sets [YPD94, DOT96]. Another alternative, in which the integer semantics of the model is verified, is also available, given the stronger assumption that the timed automaton is closed and diagonal-free.

We consider adaptations of all three methods to probabilistic timed automata. The rest of this section is divided into three parts: the first explores the verification of the abstract probabilistic timed automaton model $I_{1}^{p}$ shown in Fig. 2 using all three methods mentioned in the previous paragraph, whereas the second part considers the verification of the integer semantics of the full protocol model Impl ${ }^{\mathrm{p}}$. Finally, the statistics obtained from the probabilistic model-checking tool PRISM are presented. 
In order to establish a technical basis for the following results, we use the probabilistic temporal logic PCTL [HaJ94, Bid95] as a formal language for the deadline property. This logic is obtained from the standard temporal logic CTL [ClE81] by replacing the standard path quantifiers $\forall$ and $\exists$ by a probabilistic quantifier Pr. For example, the PCTL formula $\operatorname{Pr}_{\geqslant \lambda}(\diamond \Phi)$ is true in a state of a probabilistic system if all adversaries assign probability of at least $\lambda$ to paths which reach a state in the future for which the subformula $\Phi$ is true. Formally, the semantics of PCTL formulae with a $\diamond$ modality can be expressed using the maximal and minimal reachability probability notation in the following way. We defined the satisfaction relation of PCTL with respect to a certain subset $\mathscr{A} \subseteq A d v$ of adversaries in the manner of [BaK98]. Let $s \in S$ be a state of a probabilistic system PS, $\sqsupseteq \in\{\geqslant,>\}, \sqsubseteq \in\{\leqslant,<\}, \lambda \in[0,1]$, and $\llbracket \Phi \rrbracket_{\mathscr{A}}$ be the set of states of PS that satisfy the PCTL formula $\Phi$ when the satisfaction relation is defined with respect to the set $\mathscr{A}$ of adversaries. Then the satisfaction relation $\models_{\mathscr{A}}$ for PCTL formulae with a $\diamond$ modality, with respect to the set of adversaries $\mathscr{A} \subseteq A d v$, is defined by:

$$
s={ }_{\mathscr{A}} \operatorname{Pr}_{\sqsupseteq \lambda}(\diamond \Phi) \Leftrightarrow \operatorname{MinProbReach}_{\mathrm{PS}}\left(s, \llbracket \Phi \rrbracket_{\mathscr{A}}\right) \sqsupseteq \lambda, \quad s \models_{\mathscr{A}} \operatorname{Pr}_{\sqsubseteq \lambda}(\diamond \Phi) \Leftrightarrow \operatorname{MaxProbReach}_{\mathrm{PS}}\left(s, \llbracket \Phi \rrbracket_{\mathscr{A}}\right) \sqsubseteq \lambda
$$

Note that we use location names such as done, and constraints on clocks, such as $y \leqslant$ deadline, as 'atomic propositions' that are true or false in a state of a probabilistic system. For a more formal description of PCTL, refer to other studies [HaJ94, Bid95, BaK98].

\subsection{Abstract Model}

\subsubsection{Forward Reachability and Symbolic States}

The forward exploration method for the verification of probabilistic timed automata proceeds by a graphtheoretic search through the location space of the model, using edges, zones (of the enabling and invariant conditions) and clock resets to compute sets of reachable states of the continuous semantics. A set of states computed at any point during the algorithm is a pair comprising of location and a zone, called a symbolic state; for any classical or probabilistic timed automaton, the number of symbolic states is finite [DaT98]. Probabilistic timed reachability properties, such as 'with probability 0.99 or greater, the system reaches a leaderelected state within 100,000 ns', can be verified by defining a 'symbolic state probabilistic system', the states of which are the generated symbolic states, and the transitions of which are derived from the probabilistic timed automaton's distributions and edges [KNS02b].

We now report on the forward reachability model construction and verification of a probabilistic deadline property of the root contention protocol within a continuous time semantics, using the probabilistic modelchecking tool PRISM in conjunction with the tool HyTecH [HHW97]. The system model is taken to be the probabilistic timed automaton $\mathrm{I}_{1}^{\mathrm{p}}$ introduced in the previous section. We proceed according to the following two steps: first we describe how the automaton $I_{1}^{p}$ is decorated with additional locations and transitions to result in a new probabilistic timed automaton $I_{1}^{p+}$ for which the deadline property on $I_{1}^{p}$ is reduced to a probabilistic reachability property on $\mathrm{I}_{1}^{\mathrm{p}+}$. Secondly, we briefly describe the state-space analysis algorithm of [KNS02b], which establishes probabilistic reachability properties of probabilistic timed automata. We also describe its implementation in the scripting language of the tool $\mathrm{HYTECH}$, its application to the probabilistic timed automaton obtained in the first step, and the way in which the results obtained are subsequently used as input to PRISM.

Step 1: Decorating the Probabilistic Timed Automaton. The property of interest requires that the system elect a leader before a certain deadline with a certain probability or greater, for all adversaries. In contrast, the forward reachability procedure of Kwiatkowska et al. [KNS02b] analyses properties considering the existence of an adversary in which a certain location is reached with a given probability or greater. The former type of property can be represented in terms of the latter given adjustments to the structure of the model $\mathrm{I}_{1}^{\mathrm{p}}$ (following precedents for classical timed automata such as Lindahl et al. [LPY98]). We add the new location deadline_exceeded (which has no outgoing edges), and, from all locations apart from done, we add a distribution over a single edge with the target location deadline_exceeded. Recall that $y$ is the clock of $I_{1}^{p}$ which measures the time elapsed since the start of system execution. The enabling condition of each edge takes the form $y \geqslant$ deadline, where deadline refers to the value of the time bound considered, and the invariant condition of every location except deadline_exceeded and done is taken in conjunction with the constraint $y \leqslant$ deadline. The result of these changes is that, if the value of $y$ reached deadline in a location other than 
done, then the model must make a transition to deadline_exceeded (or done, if an enabling condition of an edge to done is satisfied) before letting time pass. We denote the new probabilistic timed automaton resulting from these changes by $\mathrm{I}_{1}^{\mathrm{p}+}$. Then the target location of the forward reachability property is taken to be deadline_exceeded. If the maximal probability with which deadline_exceeded can possibly (existentially) be reached is $\lambda$, then $1-\lambda$ will be the maximal probability with which deadline_exceeded will inevitably (universally) be avoided.

We now establish the correctness of this transformation by formalising the concepts using PCTL. Note that the forward reachability procedure can verify generic PCTL properties of the form $\operatorname{Pr}_{\sqsubseteq \lambda}(\diamond \Phi)\left(\right.$ or $\left.\neg \operatorname{Pr}_{\sqsubseteq \lambda}(\diamond \Phi)\right)$, where $\sqsubseteq \in\{<, \leqslant\}, \lambda \in[0,1]$, and $\Phi$ is a PCTL formula which does not have a subformula featuring a probabilistic quantifier Pr. We can represent the universal and existential properties described above in the syntax of PCTL: the property 'for all adversaries, the probability of reaching the location done within deadline nanoseconds is $\lambda_{1}$ or greater' is written as the PCTL formula $\operatorname{Pr}_{\geqslant}(\diamond$ (done $\wedge y \leqslant$ deadline $)$ ). Similarly, the property 'there exists an adversary such that the probability of reaching the location deadline_exceeded is $\lambda_{2}$ or greater' is written as $\neg \operatorname{Pr}_{<\lambda_{2}}(\diamond$ deadline_exceeded) (paraphrased as 'it is not true that all adversaries reach deadline_exceeded with probability strictly less than $\lambda_{2}$ ').

For an arbitrary PCTL formula $\Phi$ and $\lambda \in[0,1]$, we have $\operatorname{Pr}_{\geqslant \lambda}(\diamond \Phi) \equiv \neg \operatorname{Pr}_{<1-\lambda}(\square \neg \Phi)$ [BaK98]. Applied to the first PCTL formula given in the previous paragraph, this gives us the equivalence:

$$
\operatorname{Pr}_{\geqslant \lambda_{1}}(\diamond(\text { done } \wedge y \leqslant \text { deadline })) \equiv \neg \operatorname{Pr}_{<1-\lambda_{1}}(\square \neg(\text { done } \wedge y \leqslant \text { deadline }))
$$

Although the probabilistic quantifier $\operatorname{Pr}_{<1-\lambda_{1}}$ is of the correct form for our probabilistic timed automata verification approach, the path formula which it quantifies over uses a $\square$ temporal modality, rather than a $\diamond$ modality, as required. However, on non-Zeno paths of $\mathrm{I}_{1}^{\mathrm{p}+}$, the formulas $\square \neg($ done $\wedge y \leqslant$ deadline) and $\diamond$ deadline_exceeded are equivalent.

Lemma 4.1. Let $\omega$ be a non-Zeno path of $\llbracket \mathrm{I}_{1}^{\mathrm{p}+} \rrbracket_{\mathbb{R}}$, and let $\mathscr{A} \subseteq A d v$. Then $\omega=_{\mathscr{A}} \square \neg($ done $\wedge y \leqslant$ deadline) if and only if $\omega={ }_{\mathscr{A}} \diamond$ deadline_exceeded.

Proof. See Appendix B.

Corollary 4.2. Let $\mathscr{A}_{n Z} \subseteq A d v$ be the set of non-Zeno adversaries of $\llbracket I_{1}^{\mathrm{p}+} \rrbracket_{\mathbb{R}}$. Then (start_start, $\left.\mathbf{0}\right) \models \mathscr{A}_{n Z}$ $\operatorname{Pr}_{<1-\lambda_{1}}(\square \neg($ done $\wedge y \leqslant$ deadline $))$ if and only if (start_start, $\left.\mathbf{0}\right) \models_{\mathscr{A}_{n Z}} \operatorname{Pr}_{<1-\lambda_{1}}(\diamond$ deadline_exceeded).

The corollary follows because, although a non-Zeno adversary may exhibit Zeno paths (for which the equivalence of Lemma 4.1 does not hold), the probability measure of such paths must be 0 . Hence, we have reduced the PCTL formula $\operatorname{Pr}_{\geqslant \lambda_{1}}(\diamond($ done $\wedge y \leqslant$ deadline $))$ to $\neg \operatorname{Pr}_{<1-\lambda_{1}}$ ( $\diamond$ deadline_exceeded) on the non-Zeno adversaries of $\mathrm{I}_{1}^{\mathrm{p}+}$. That is, we have converted a formula concerning the reachability of a certain set of states with a certain probability or greater into a formula concerning the reachability of a different set of states with a certain probability or less.

Not all adversaries of $\mathrm{I}_{1}^{\mathrm{p}+}$ (and indeed $\mathrm{I}_{1}^{\mathrm{p}}$ ) are non-Zeno, because an adversary may choose to advance time in a Zeno, or convergent manner (for example, letting 0 nanoseconds elapse in all time transitions performed from some point onwards). This would be problematic for our analysis if the set of adversaries which reach the target location deadline_exceeded with the maximal probability are all Zeno. The following lemma shows that this is not the case: more precisely, we show that, for any Zeno adversary of $\mathrm{I}_{1}^{\mathrm{p}+}$, there exists a non-Zeno adversary which can reach the location deadline_exceeded with an equal or greater probability.

Lemma 4.3. Let $A$ be a Zeno adversary of $\llbracket I_{1}^{\mathrm{p}+} \rrbracket_{\mathbb{R}}$. Then there exists a non-Zeno adversary $A_{n Z}$ of $\llbracket I_{1}^{\mathrm{p}+} \rrbracket_{\mathbb{R}}$ such that:

$$
\operatorname{ProbReach}^{A_{n Z}}\left(\left(\text { start_start, 0), deadline_exceeded) } \geqslant \operatorname{ProbReach}^{A}((\text { start_start, 0), deadline_exceeded })\right.\right.
$$

Proof. See Appendix C.

Step 2: Applying Forward Reachability to Probabilistic Timed Automata. The second step involves the computation of the relevant symbolic states, together with information concerning transitions made between such symbolic states, using the tool HYTECH. As this tool is equipped with a simple programming language in which the symbolic states of a classical timed automaton serve as the basic data structure, it permits us to implement the forward reachability algorithm of [KNS02b], given that a number of auxiliary, discrete, integer-valued 
variables are added to a classical timed automaton representation of $\mathrm{I}_{1}^{\mathrm{p}+}$ so that sufficient information concerning the probabilistic transition relation between the symbolic states is maintained. Firstly, the additional variable edge is used to record the edge of the classical timed automaton that was last traversed; this is achieved by assigning to each edge of the model a different value, and then setting the variable edge to this value upon traversal of the relevant edge. Secondly, the variable number is used to uniquely number the symbolic states that are generated by the program; this is to distinguish sets of states which may have the same location, but, because they belong to different symbolic states, may correspond to different probabilities of reaching the target location deadline_exceeded. Finally, when a new symbolic state is generated (recall that this occurs when the effect of a discrete transition is applied to a previously generated symbolic state), the variable source is set to the value of number corresponding to the source symbolic state. Note that a symbolic state may have more than one value of source, meaning that it may be reached from more than one symbolic state in a single discrete transition.

We then perform probabilistic model checking on the resulting 'symbolic state probabilistic system', the states of which correspond to the symbolic states generated by the previous step, and the transitions of which are derived from the values of edge and source in each symbolic state, against a PCTL property which refers to the maximum probability of reaching any symbolic state with the location component deadline_exceeded.

Although it is established [KNS02b] that, in general, the forward reachability process described above is only guaranteed to compute an upper bound on the reachability probability in the probabilistic timed automaton, for certain classes of systems, the technique will result in the computation of the exact probability; indeed, the probabilistic timed automaton $\mathrm{I}_{1}^{\mathrm{p}+}$ falls into this class. Rather than present a formal proof, we provide an experimental validation of this result by verifying that the probabilities obtained using the forward reachability method agree with those obtained on the region graph and the probabilistic timed automaton with integer semantics.

\subsubsection{Region Graph}

The second approach taken for the verification of the abstract probabilistic timed automaton model $\mathrm{I}_{1}^{\mathrm{p}}$ involves the definition of a finite state region graph. Such a graph is obtained from a finite partition of the clock valuation space first defined in the context of classical timed automata in Alur and Dill [A1D94]. The region graph is defined using the combination of this partition with the locations and transitions of the model. It is shown in [KNS02b] that the region graph of a probabilistic timed automaton takes the form of a probabilistic system which can be used to verify PCTL properties. Hence, we can use the region graph of $\mathrm{I}_{1}^{\mathrm{p}}$ for probabilistic model checking of the property $\operatorname{Pr}_{\geqslant \lambda_{1}}(\diamond($ done $\wedge y \leqslant$ deadline $))$ directly. For efficiency, we combine all states corresponding to the bound deadline being exceeded by the clock $y$ into a single state.

This region graph is then encoded directly into the system description language of PRISM in anticipation of probabilistic model checking. The description language is state-based, where a state of the model corresponds to a valuation of a finite set of finite-domain variables, and where the state transitions are obtained by guarded commands which establish a relationship between the values of the state variables before and after a transition. We use six variables to encode the region graph: one for the location component of the region, two for the integer values of each of the clocks $x$ and $y$, two to record whether the fractional part of the clocks is 0 or not, and one to record the order on the fractional parts of the two clocks.

\subsubsection{Integer Semantics}

The final technique for the verification of the abstract probabilistic timed automaton $I_{1}^{p}$ concerns the use of its integer semantics, defined in Definition 2.4. As in the previous two subsections, we obtain a finite state probabilistic system which can be model checked using PRISM. The encoding of the integer-semantics probabilistic system into the system description language of PRISM involves the use of three variables: one to denote the location component of the state, and two to denote the integer values of the clocks.

As the probabilistic timed automaton $\mathrm{I}_{1}^{\mathrm{p}}$ is closed and diagonal-free, Theorem 2.5 holds, and therefore the minimum probability of reaching a state with location component done before the deadline is the same in the continuous and integer semantics. 
Table 1. Verification results for the abstract model $I_{1}^{p}$ with the wire delay set to $360 \mathrm{~ns}$.

\begin{tabular}{|c|c|c|c|c|c|c|c|c|c|c|}
\hline \multirow[t]{2}{*}{ Deadline (ns) } & \multicolumn{3}{|c|}{ Forward reachability } & \multicolumn{3}{|c|}{ Region graph } & \multicolumn{3}{|c|}{ Integer semantics } & \multirow[t]{2}{*}{ Probability } \\
\hline & States & Iters. & Time (s) & States & Iters. & Time (s) & States & Iters. & Time (s) & \\
\hline 2,000 & 48 & 13 & 0.06 & 423,016 & 670 & 13.2 & 68,185 & 169 & 1.31 & 0 \\
\hline 2,500 & 68 & 26 & 0.07 & 652,890 & 743 & 18.9 & 104,333 & 207 & 4.08 & 0.5 \\
\hline 3,000 & 72 & 26 & 0.09 & 900,390 & 1,157 & 50.2 & 143,133 & 293 & 3.86 & 0.5 \\
\hline 3,500 & 103 & 31 & 0.11 & $1,147,890$ & 1,230 & 68.2 & 181,933 & 331 & 5.43 & 0.625 \\
\hline 4,000 & 137 & 33 & 0.13 & $1,395,390$ & 1,485 & 119 & 220,733 & 375 & 7.72 & 0.625 \\
\hline 4,500 & 142 & 38 & 0.16 & $1,642,890$ & 1,717 & 139 & 259,533 & 455 & 11.7 & 0.78125 \\
\hline 5,000 & 183 & 38 & 0.19 & $1,890,390$ & 1,972 & 175 & 298,333 & 499 & 16.1 & 0.78125 \\
\hline 5,500 & 218 & 40 & 0.22 & $2,137,890$ & 2,131 & 228 & 337,133 & 541 & 24.9 & 0.84375 \\
\hline 6,000 & 234 & 45 & 0.24 & $2,385,390$ & 2,300 & 265 & 375,933 & 581 & 22.5 & 0.851562 \\
\hline 7,000 & - & - & - & $2,880,390$ & 2,787 & 374 & 453,533 & 705 & 29.8 & 0.908203 \\
\hline 8,000 & - & - & - & $3,375,390$ & 3,115 & 519 & 531,133 & 789 & 39.6 & 0.939453 \\
\hline 9,000 & - & - & - & $3,870,390$ & 3,602 & 659 & 608,733 & 913 & 51.5 & 0.961914 \\
\hline 10,000 & - & - & - & $4,365,390$ & 3,930 & 843 & 686,333 & 995 & 62.1 & 0.974731 \\
\hline 100,000 & - & - & - & $48,915,390$ & 12,229 & 6,007 & $7,670,333$ & 3,097 & 599 & 0.999996 \\
\hline $1,000,000$ & - & - & - & - & - & - & $77,510,333$ & 3,097 & 589 & 0.999996 \\
\hline$\infty$ & - & - & - & 1,542 & 1,492 & 1.25 & 776 & 752 & 0.51 & 1 \\
\hline
\end{tabular}

Table 2. Verification results for the abstract model $I_{1}^{\mathrm{p}}$ with the wire delay set to $30 \mathrm{~ns}$.

\begin{tabular}{|c|c|c|c|c|c|c|c|c|c|c|}
\hline \multirow[t]{2}{*}{ Deadline (ns) } & \multicolumn{3}{|c|}{ Forward Reachability } & \multicolumn{3}{|c|}{ Region graph } & \multicolumn{3}{|c|}{ Integer semantics } & \multirow[t]{2}{*}{ Probability } \\
\hline & States & Iters. & Time $(\mathrm{s})$ & States & Iters. & Time (s) & States & Iters. & Time $(\mathrm{s})$ & \\
\hline 1,500 & 32 & 8 & 0.02 & 57,903 & 1 & 0.04 & 7,235 & 1 & 0.02 & 0 \\
\hline 2,000 & 51 & 11 & 0.04 & 85,638 & 677 & 2.31 & 14,868 & 174 & 0.36 & 0.5 \\
\hline 2,500 & 71 & 11 & 0.05 & 144,450 & 684 & 5.09 & 24,389 & 174 & 0.81 & 0.5 \\
\hline 3,000 & 71 & 14 & 0.07 & 222,090 & 1,032 & 9.94 & 36,410 & 265 & 0.94 & 0.625 \\
\hline 3,500 & 101 & 14 & 0.11 & 319,852 & 1,387 & 29.8 & 52,108 & 356 & 4.09 & 0.78125 \\
\hline 4,000 & 131 & 17 & 0.15 & 433,018 & 1,387 & 35.2 & 69,791 & 356 & 4.69 & 0.78125 \\
\hline 4,500 & 131 & 20 & 0.16 & 563,570 & 1,742 & 67.8 & 90,212 & 447 & 8.80 & 0.851563 \\
\hline 5,000 & 171 & 20 & 0.17 & 715,390 & 1,742 & 92.5 & 114,001 & 447 & 12.3 & 0.851563 \\
\hline 5,500 & 202 & 23 & 0.19 & 880,754 & 2,097 & 122 & 139,817 & 538 & 22.0 & 0.908203 \\
\hline 6,000 & 211 & 23 & 0.21 & $1,065,154$ & 2,398 & 193 & 168,567 & 611 & 27.2 & 0.931641 \\
\hline 7,000 & - & - & - & $1,455,737$ & 2,807 & 248 & 229,085 & 720 & 40.6 & 0.962036 \\
\hline 8,000 & - & - & - & $1,851,737$ & 3,162 & 293 & 290,185 & 811 & 56.8 & 0.975494 \\
\hline 9,000 & - & - & - & $2,247,737$ & 3,517 & 439 & 351,285 & 902 & 76.7 & 0.984383 \\
\hline 10,000 & - & - & - & $2,643,737$ & 3,872 & 612 & 412,385 & 993 & 103 & 0.989970 \\
\hline 100,000 & - & - & - & $38,283,737$ & 9,985 & 1,059 & $5,911,385$ & 2,536 & 736 & 0.999996 \\
\hline $1,000,000$ & - & - & - & - & - & - & $60,901,385$ & 2,536 & 757 & 0.999996 \\
\hline$\infty$ & - & - & - & 1,212 & 1,360 & 1.11 & 611 & 686 & 0.52 & 1 \\
\hline
\end{tabular}

\subsubsection{Results}

The results obtained from verifying the abstract model are shown in Tables 1 and 2. We include the verification results for the case in which the communication delay along the wires between the two nodes is $360 \mathrm{~ns}$ in Table 1, and $30 \mathrm{~ns}$ in Table 2. In both tables, the left-most column shows the deadline used in the property, and the right-most column shows the minimum probability with which the system reaches a leader-elected state before the deadline (the same probability was computed by all three methods). The results reflect the obvious fact that increasing the value of the deadline has the effect of increasing the probability of a timely leader election; intuitively, the greater the deadline, the 'more chances' the system has of flipping different results for each node (therefore electing a leader). Observe that the rate of increase both in the number of computed symbolic states and the probability proceeds in 'jumps' as the deadline increases. In particular, the probability of satisfying the required property is dependent on how many protocol restarts (and therefore coin flips) the system can perform before expiration of the deadline. Although the generation of probabilities for low values of the deadline (particularly 3000 or less) is trivial, the probability computation becomes more involved for greater deadlines.

For all three techniques (forward reachability, region graph and integer semantics), the sub-columns marked 'States' give the number of states of the probabilistic system which is taken as input to PRISM, 
the sub-columns marked 'Iters.' give the number of iterations taken by PRISM to compute the probability, and the sub-columns marked 'Time' give the time in seconds taken by PRISM for the computation. Observe that the probability shown in the right-most column of the table is actually one minus the maximum of the existential reachability property computed by PRISM using the forward reachability method (as we verify the dual of the actual formula we are interested in). As expected, the probabilities computed for all three methods agree. A dash '-' denotes that verification was not attempted.

The symbolic states generated by the forward reachability algorithm, as implemented in HYTECH, form the states of the probabilistic system taken as input to PRISM. We do not include time and memory statistics for HYTECH as, in general, the execution of the forward reachability algorithm implementation periodically required termination and subsequent re-execution in order to avoid excessive memory consumption (for higher deadlines, this was achieved using a shell script). As a guide, the symbolic states for the deadline of $6000 \mathrm{~ns}$ took approximately 24 hours to obtain. This performance bottleneck made the verification of greater deadlines impractical. In contrast to this poor performance, observe that the state spaces which are generated by the forward reachability method are significantly smaller (by an order of magnitude of $10^{4}$ compared to the region graph technique, and by an order of $10^{3}$ compared to the integer semantics technique). Deadlines of up to $100,000 \mathrm{~ns}$ and $1,000,000 \mathrm{~ns}$ are within reach of the capabilities of PRISM using the region graph and the integer semantics, respectively, although it should be noted that PRISM is designed to handle large state spaces using efficient data structures such as Multi-Terminal Binary Decision Diagrams (MTBDDs) [CFM93]. Using the integer semantics of $I_{1}^{p}$ results in performance gains over the region-based approach (the state spaces generated are just over six times smaller than those obtained from the region graph), which is explained by observing that the information encoded into the integer semantics is a strict subset of that encoded into the region graph.

In addition to the verification times given above, the tool PRISM also involves a model construction phase, during which the system description is parsed, converted into an MTBDD representation, and subjected to an efficient, BDD-based state-space exploration procedure to find the reachable states of the system [dKN00]. As a guide, for a deadline of 100,000, the model construction time for the region graph is approximately 20 minutes, compared to 14 minutes for the integer semantics model. For more information, including model construction times for all deadlines, see the web site [Pri].

As PRISM computes the probability of property satisfaction using an iterative technique, a termination criterion is given by a sufficiently small $\epsilon$, where the iteration terminates if the relative difference of successively computed probabilities is less than $\epsilon$. For this case study, we set $\epsilon=10^{-6}$. Hence, although the probabilities computed using the integer semantics for deadlines of 100,000 and 1,000,000 ns are identical, this should not be interpreted as indicating that the probabilities have converged. Indeed, the final row of the table gives the performance statistics of the verification of the property $\operatorname{Pr}_{\geqslant \lambda_{3}}(\diamond($ done $) \wedge y \leqslant \infty) \equiv \operatorname{Pr}_{\geqslant \lambda_{3}}(\diamond($ done $))$ on the region graph and integer semantics (such a property is out of the scope of the forward reachability procedure). We perform verification on a simplified model in which the clock $y$, used to measure global time, is not represented. Use of PRISM establishes that this property is satisfied even for $\lambda_{3}=1$, and therefore we conclude that the probability of electing a leader converges to 1 as the deadline approaches $\infty$. The efficiency of PRISM for this property is due to the fact that a graph-theoretic pre-computation procedure which calculates the states satisfying formulae with probability 1 is used in the model-checking phase.

\subsection{Full Model}

In this section, we report on the application of the most efficient verification method employed in the previous section, that concerning integer semantics, to the full probabilistic timed automaton $\operatorname{Imp} 1^{p}$ of the root contention protocol. As with the case of $I_{1}^{p}$ in the previous section, verification on the integer semantics is sufficient because $\operatorname{Imp} 1^{p}$ is a closed, diagonal-free probabilistic timed automaton, and, by Theorem 2.5 , the minimum probability of reaching a state with a leader-elected location before the deadline is the same for the continuous and integer semantics of $\mathrm{Impl}^{\mathrm{p}}$. We do not attempt the region graph technique, as the state space generated by this method is strictly greater than that for the integer semantics.

Rather than construct the integer semantics $\llbracket I m p 1^{p} \rrbracket_{\mathbb{N}}$ of the composed probabilistic timed automaton $\operatorname{Impl}{ }^{\mathrm{p}}=$ Node $_{1}^{\mathrm{p}} \|$ Wire $_{1} \|$ Wire $_{2} \|$ Node $_{2}^{\mathrm{p}}$, we elected to define the integer semantics of each of the four components of $\operatorname{Impl}{ }^{\mathrm{p}}$, and then use the parallel composition facility of PRISM to generate the probabilistic system:

$$
\llbracket \operatorname{Node}_{1}^{\mathrm{p}} \rrbracket_{\mathbb{N}} \| \llbracket \text { Wire } \rrbracket_{1}\left\|\llbracket \operatorname{Wire}_{2} \rrbracket_{\mathbb{N}}\right\| \llbracket \operatorname{Node}_{2}^{\mathrm{p}} \rrbracket_{\mathbb{N}}
$$


Table 3. Verification results for the full model $\operatorname{Impl}{ }^{\mathrm{p}}$.

\begin{tabular}{|c|c|c|c|c|c|c|c|c|}
\hline \multirow[t]{2}{*}{ Deadline (ns) } & \multicolumn{4}{|c|}{ Wire delay of $360 \mathrm{~ns}$} & \multicolumn{4}{|c|}{ Wire delay of $30 \mathrm{~ns}$} \\
\hline & States & Iters. & Time (s) & Probability & States & Iters. & Time (s) & Probability \\
\hline 1,500 & $2,426,116$ & 48 & 6.34 & 0 & 29,631 & 15 & 0.35 & 0 \\
\hline 2,000 & $6,719,773$ & 50 & 20.2 & 0 & 80,980 & 179 & 4.23 & 0.5 \\
\hline 2,500 & $14,089,691$ & 212 & 51.7 & 0.5 & 143,394 & 185 & 7.58 & 0.5 \\
\hline 3,000 & $23,319,148$ & 213 & 287 & 0.5 & 213,805 & 275 & 12.9 & 0.625 \\
\hline 3,500 & $33,789,641$ & 341 & 621 & 0.625 & 328,547 & 371 & 22.8 & 0.78125 \\
\hline 4,000 & $44,366,235$ & 341 & 7,325 & 0.625 & 434,364 & 371 & 29.3 & 0.78125 \\
\hline 4,500 & $54,973,279$ & 470 & 11,338 & 0.78125 & 572,437 & 467 & 40.7 & 0.851563 \\
\hline 5,000 & $65,586,679$ & 470 & 24,556 & 0.78125 & 731,109 & 467 & 61.7 & 0.851563 \\
\hline 5,500 & $76,200,079$ & 552 & 23,229 & 0.84375 & 899,798 & 563 & 71.5 & 0.908203 \\
\hline 6,000 & $86,813,479$ & 599 & 35,293 & 0.851562 & $1,093,658$ & 631 & 114 & 0.931641 \\
\hline 7,000 & $108,040,279$ & 728 & 51,028 & 0.908203 & $1,499,591$ & 755 & 132 & 0.962036 \\
\hline 8,000 & $129,267,079$ & 810 & 58,504 & 0.939453 & $1,915,291$ & 851 & 209 & 0.975494 \\
\hline 9,000 & $150,493,879$ & 939 & 73,829 & 0.961914 & $2,330,991$ & 947 & 253 & 0.984383 \\
\hline 10,000 & $171,720,679$ & 1,021 & 95,000 & 0.974731 & $2,746,691$ & 1,043 & 296 & 0.989970 \\
\hline$\infty$ & 212,268 & 844 & 336 & 1 & 4,157 & 712 & 36.2 & 1 \\
\hline
\end{tabular}

As it was established that $\llbracket \operatorname{Imp} I^{\mathrm{p}} \rrbracket_{\mathbb{N}}$ is isomorphic to $\llbracket$ Node $_{1}^{\mathrm{p}} \rrbracket_{\mathbb{N}} \| \llbracket$ Wire ${ }_{1} \rrbracket_{\mathbb{N}} \| \llbracket$ Wire $\rrbracket_{2} \rrbracket_{\mathbb{N}} \| \llbracket$ Node ${ }_{2}^{\mathrm{p}} \rrbracket_{\mathbb{N}}$ in Section 2.2.1, and as isomorphism is stronger than (probabilistic) bisimulation, which preserves all PCTL formulae on probabilistic systems [SeL95], we conclude that the two systems satisfy the same PCTL formulae, including the formula $\operatorname{Pr}_{\geqslant \lambda}(\diamond($ done $\wedge y \leqslant$ deadline $))$ requiring that 'for all adversaries, the probability of electing a leader

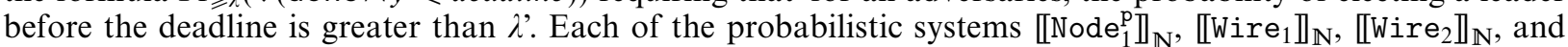
$\llbracket \operatorname{Node}_{2}^{\mathrm{p}} \rrbracket_{\mathbb{N}}$ is encoded as a separate 'module', with its own state variables, within the language of PRISM. For example, the module for $\llbracket N o d e_{1}^{p} \rrbracket_{N}$ features two variables: one corresponding to the location component of the state, and the other recording the current value of the clock of Node ${ }_{1}^{\mathrm{p}}$. Finally, we also include a module recording global time using a single variable.

The results obtained from generating the integer semantics of the full model are given in Table 3 , both for a communication delay of $360 \mathrm{~ns}$ and for $30 \mathrm{~ns}$. Note that the automatic model construction process generally took a small number of minutes, in addition to the computation time given in the table (see the web page [Pri] for full details). We observe that the ratio of the number of states obtained from this method to the number of states obtained from the integer semantics of the abstract model $\mathrm{I}_{1}^{\mathrm{p}}$ increases as the deadline increases (from a ratio of approximately 100 for the deadline $2000 \mathrm{~ns}$, to approximately 250 for the deadline 10,000 ns), although at a decreasing rate; therefore, the abstract model $\mathrm{I}_{1}^{\mathrm{p}}$ scales better with regard to the largest numerical constant of the system. Note also that the probabilities obtained using the full model agree with those obtained from verification on the abstract model.

Although the utility of integer semantics is limited to closed, diagonal-free classical and probabilistic timed automata, in this case such semantics have allowed us to verify high deadlines without the need for abstraction. We envisage that the combination of integer semantics and abstraction may lead to successful verification of more complex probabilistic real-time systems.

\section{Conclusions}

We have considered an approach to the formal specification of both timed and probabilistic aspects of the root contention protocol of IEEE 1394 using probabilistic timed automata, and have investigated the way in which the tools PRISM and HYTECH may be used to automatically verify the resulting model against deadline properties. The properties that we consider are closely related to the property 'a leader is eventually chosen', whereas we do not consider safety properties such as 'only one leader is chosen'. The verification used a variety of techniques, including high-level abstraction and methods for the construction of finite-state probabilistic systems using explorative algorithms, region equivalence and non-standard integer semantics. The abstraction method considered was originally developed [StV99] as one step of a manual proof; we envisage that automatic, probabilistic abstraction methods, for example D'Argenio et al. [DJJ01], could also be applied to improve the efficiency of probabilistic timed automata model checking.

We consider probabilistic timed automata to be particularly appropriate for the specification of the nodes involved in the root contention protocol, together with their communication media. Probabilistic timed 
automata can express a variety of notions which are important in representing the nodes and wires, such as the probabilistic coin-flip, the communication between the components, and the non-deterministic choice of time delays (within given short or long waiting intervals) in a formal yet concise way. The timing parameters from the updated IEEE 1394a standard were used in the models, although observe that it is straightforward to change the values of the parameters and rerun the experiments, as we did for the wire propagation delay. Such a change of parameters results in the loss of the work performed in previous analyses, although, because the analysis procedure is automatic, this involves no significant manual effort. We consider probabilistic model checking to be an appropriate technique for the analysis of the root contention protocol, as manual analysis is notoriously prone to human error, even for simple systems.

When evaluating the total time taken to obtain a solution via our analysis, we should consider that both the modelling and analysis of Simons and Stoelinga [SiS01] required approximately two person months. As we use the classical timed automata models (and some of the results) of Simons and Stoelinga [SiS01] as a basis for our probabilistic models, we should regard this as the time taken also to derive our models. Subsequent translation of these models into the HYTECH and PRISM description languages required a small number of hours (with the region graph models being the most difficult to encode, as detailed understanding of its partitioning method is necessary). We note that, given knowledge of probabilistic systems and classical timed automata, the textual system description languages of PRISM and HYTECH are easy to learn, requiring only a number of hours of effort. For an average programmer, or a person not literate in formal methods, the task of obtaining sufficient knowledge of the formalisms may be more challenging, but we nevertheless estimate this to be possible within a week. In terms of analysis, as noted in Section 4, the forward reachability constructions implemented in HyTECH took approximately 24 hours for the higher values of the deadline considered, whereas model checking the resulting probabilistic systems using PRISM required seconds; similarly, model checking using PRISM alone on the region graph and the integer semantics required at most 27 hours for the full model $\mathrm{Impl} \mathrm{l}^{\mathrm{p}}$. Finally, the proofs of correctness for the minimal and maximal reachability probabilities obtained via the integer semantics, and the results concerning non-Zenoness for the forward reachability analysis, each took a small number of days.

In terms of future work, our models could be used as a basis for the automatic computation of the expected time to leader election; it would therefore be of interest to formalise such a notion in the context of probabilistic timed automata, and to relate it to other precedents in the probabilistic model checking literature, for example de Alfaro [deA97]. One aspect that is missing from the forward reachability approach is an automatic method for verifying that the probability of the system electing a leader within a deadline of infinity is 1 . This requires a thorough investigation into such a class of probabilistic 'liveness' properties, which cannot be analysed by use of the reachability algorithm of Kwiatkowska et al. [KNS02b]. Although manual proof methods dealing with such expected-time and probabilistic liveness properties have been known for some time, in Kozen [Koz81] and Hart et al. [HSP83] respectively, we reiterate that such proof methods are prone to error, and that there is a chronic need for automatic, model-checking methods for such properties.

The verification of the root contention protocol was carried out as part of an ongoing activity concerning the practical implementation of model-checking methods for probabilistic timed automata. One lesson learned was that, although the size of the generated state spaces can be handled easily by the tool PRISM, the high memory usage of our algorithm implementation in НYТЕсH proved to be the bottleneck of the analysis, and meant that we were able to perform verification of only short deadlines. This is to be expected, as НүТЕсн is a flexible tool which can be applied to a superclass of timed automata, including hybrid automata and parameterised systems, and for which efficient data structures have not been implemented; in contrast, PRISM uses the compact data structure of MTBDDs to represent probabilistic systems. Indeed, the forward reachability algorithm has been implemented recently in the classical timed automata tool KRONOS [DOT96], for which efficient data structures to represent symbolic states have been developed, with results that far surpass those obtained with HyTECH [DKN02].

\section{Acknowledgements}

This work was supported in part by the EPSRC grants GR/M04617 and GR/N22960, and by the EU within the DepAuDE project IST-2001-25434. 


\section{References}

[ACD91] Alur, R., Courcoubetis, C., and Dill, D. L,: Model-checking for probabilistic real-time systems. In J. Leach Albert, B. Monien and M. Artalejo Rodríguez, editors, Proceedings of the 18th International Conference on Automata, Languages and Programming (ICALP'91), volume 510 of LNCS, pages 115-136. Springer-Verlag, 1991.

[AlD94] Alur, R. and Dill, D. L.: A theory of timed automata. Theoretical Computer Science, 126(2):183-235, 1994.

[AIK95] Alur, R., Itai, A., Kurshan, R. and Yannakakis, M.: Timing verification by successive approximation. Information and Computation, 18(1):142-157, 1995.

[AMP98] Asarin, E., Maler, O. and Pnueli, A.: On discretization of delays in timed automata and digital circuits. In Sangiorgi and de Simone [Sad98], pages 470-484.

[BaK98] Baier, C. and Kwiatkowska, M. Z.: Model checking for a probabilistic branching time logic with fairness. Distributed Computing, 11(3):125-155, 1998.

[Bey01] Beyer, D.: Improvements in BDD-based reachability analysis of timed automata. In J. N. Oliveira and P. Zave, editors, Proceedings of the 10th International Symposium of Formal Methods Europe (FME 2001), volume 2021 of LNCS, pages 318-343. Springer-Verlag, 2001.

[BKH99] Baier, C., Katoen, J.-P. and Hermanns, H.: Approximate symbolic model checking of continuous-time Markov chains. In J. Baeten and S. Mauw, editors, Proceedings of the 10th International Conference on Concurrency Theory (CONCUR'99), volume 1664 of $L N C S$, pages 142-162. Springer-Verlag, 1999.

[Bid95] Bianco, A. and de Alfaro, L.: Model checking of probabilistic and nondeterministic systems. In P. S. Thiagarajan, editor, Proceedings of the International Conference on the Foundations of Software Technology and Theoretical Computer Science (FSTTCS'95), volume 1026 of LNCS, pages 499-513. Springer-Verlag, 1995.

[BMT99] Bozga, M., Maler, O. and Tripakis, S.: Efficient verification of timed automata using dense and discrete time semantics. In L. Pierre and T. Kropf, editors, Proceedings of the 11th Advanced Research Working Conference on Correct Hardware Design and Verification Methods (CHARME'99), volume 1703 of LNCS, pages 125-141. Springer-Verlag, 1999.

[BST00] Bandini, G., Spelberg, R. L. and Toetenel, W. J.: Parametric verification of the IEEE 1394a root contention protocol using LPMC. In Proceedings of the 7th International Conference on Real-Time Computing Systems and Applications (RTCSA 2000), pages 207-214. IEEE Computer Society Press, 2000.

[ClE81] Clarke, E. M. and Emerson, E. A.: Design and synthesis of synchronization skeletons using branching time temporal logic. In D. Kozen, editor, Proceedings of the Workshop on Logics of Programs, volume 131 of LNCS, pages 52-71. Springer-Verlag, 1981.

[CFM93] Clarke, E. M., Fujita, M., McGeer, P., McMillan, K., Yang, J. and Zhao, X.: Multi-terminal binary decision diagrams: an efficient data structure for matrix representation. In Proceedings of the International Workshop on Logic Synthesis (IWLS'93), pages 6a:1-15, 1993. Also available in Formal Methods in System Design, 10(2/3):149-169, 1997.

[CoS01] Collomb-Annichini, A. and Sighireanu, M.: Parameterized reachability analysis of the IEEE 1394 root contention protocol using TReX. In P. Pettersson and S. Yovine, editors, Proceedings of the Workshop on Real-Time Tools (RT-TOOLS 2001), 2001 .

[deA97] de Alfaro, L.: Formal Verification of Probabilistic Systems. PhD thesis, Stanford University, 1997.

[deA98] de Alfaro, L.: Stochastic transition systems. In Sangiorgi and de Simone [Sad98], pages 423 - 438.

[DAr99] D'Argenio, P. R.: Algebras and Automata for Timed and Stochastic Systems. PhD thesis, Department of Computer Science, University of Twente, 1999.

[dKN00] de Alfaro, L., Kwiatkowska, M. Z., Norman, G., Parker, D. and Segala, R.: Symbolic model checking of concurrent probabilistic processes using MTBDDs and the Kronecker representation. In S. Graf and M. Schwartzbach, editors, Proceedings of the 6th International Conference on Tools and Algorithms for the Construction and Analysis of Systems (TACAS 2000), volume 1785 of LNCS, pages 395-410. Springer-Verlag, 2000.

[Der70] Derman, C.: Finite-State Markovian Decision Processes. Academic Press, 1970.

[DJJ01] D’Argenio, P. R., Jeannet, B., Jensen, H. E. and Larsen, K. G.: Reachability analysis of probabilistic systems by successive refinements. In L. de Alfaro and S. Gilmore, editors, Proceedings of the Joint PAPM-PROBMIV 2001 Workshop, volume 2165 of LNCS, pages 39-56. Springer-Verlag, 2001.

[DKN02] Daws, C., Kwiatkowska, M. Z. and Norman, G.: Automatic verification of IEEE 1394 root contention protocol with KRONOS and PRISM. In R. Cleaveland and H. Garavel, editors, Proceedings of the 7th International Workshop on Formal Methods for Industrial Critical Systems (FMICS 2002), pages 107-122, 2002. To appear in Electronic Notes in Theoretical Computer Science.

[DOT96] Daws, C., Olivero, A., Tripakis, S. and Yovine, S.: The tool Kronos. In R. Alur, T. A. Henzinger and E. D. Sontag, editors, Hybrid Systems III: Verification and Control, volume 1066 of LNCS, pages 208-219. Springer-Verlag, 1996.

[DaT98] Daws, C. and Tripakis, S.: Model-checking of real-time reachability properties using abstractions. In Steffen [Ste98], pages $313-329$.

[DaY95] Daws, C. and Yovine, S.: Two examples of verification of multirate timed automata with KRoNos. In A. Burns, Y.-H. Lee and K. Ramamritham, editors, Proceedings of the 16th IEEE Real-Time Systems Symposium (RTSS'95), pages 66-75. IEEE Computer Society Press, 1995.

[FiS01] Fidge, C. and Shankland, C.: But what if I don't want to wait forever? In S. Maharaj, J. Romijn and C. Shankland, editors, Proceedings of the International Workshop on Application of Formal Methods to IEEE 1394 Standard, 2001.

[HaJ94] Hansson, H. and Jonsson, B.: A logic for reasoning about time and reliability. Formal Aspects of Computing, 6(5):512-535, 1994.

[HHW95] Henzinger, T. A., Ho, P.-H. and Wong-Toi, H.: A user guide to HyTech. In E. Brinksma, W. R. Cleaveland, K. G. Larsen, T. Margaria and B. Steffen, editors, Proceedings of the 1st International Workshop on Tools and Algorithms for the Construction and Analysis of Systems (TACAS'95), volume 1019 of LNCS, pages 41-71. Springer-Verlag, 1995.

[HHW97] Henzinger, T. A., Ho, P.-H. and Wong-Toi, H.: HyTech: a model checker for hybrid systems. Software Tools for Technology Transfer, 1(1+2):110-122, 1997. 
[HMP92] Henzinger, T. A., Manna, Z. and Pnueli, A.: What good are digital clocks? In W. Kuich, editor, Proceedings of the 19th International Colloquium on Automata, Languages, and Programming (ICALP'92), volume 623 of LNCS, pages 545-558. Springer-Verlag, 1992.

[HRS01] Hune, T. S., Romijn, J. M. T., Stoelinga, M. I. A. and Vaandrager, F. W.: Linear parametric model checking of timed automata. In T. Margaria and W. Yi, editors, Proceedings of the 7th Conference on Tools and Algorithms for the Construction and Analysis of Systems (TACAS 2001), volume 2031 of LNCS, pages 189-203. Springer-Verlag, 2001.

[HSP83] Hart, S., Sharir, M. and Pnueli, A.: Termination of probabilistic concurrent programs. ACM Transactions on Programming Languages and Systems, 5(3):356-380, 1983

[Kat99] Katoen, J.-P., editor: Proceedings of the 5th International AMAST Workshop on Real-Time and Probabilistic Systems (ARTS'99), volume 1601 of LNCS. Springer-Verlag, 1999.

[KNP02] Kwiatkowska, M. Z., Norman, G. and Parker, D.: PRISM: probabilistic symbolic model checker. In T. Field, P. G. Harrison, J. Bradley and U. Harder, editors, Proceedings of the 12th International Conference on Computer Performance Evaluation, Modelling Techniques and Tools (TOOLS 2002), volume 2324 of LNCS, pages 200-204. Springer-Verlag, 2002.

[KNS02a] Kwiatkowska, M. Z., Norman, G. and Sproston, J.: Probabilistic model checking of the IEEE 802.11 wireless local area network protocol. In H. Hermanns and R. Segala, editors, Proceedings of the Joint PAPM-PROBMIV 2002 Workshop, volume 2399 of $L N C S$, pages 169-187. Springer-Verlag, 2002

[KNS02b] Kwiatkowska, M. Z., Norman, G., Segala, R. and Sproston, J.: Automatic verification of real-time systems with discrete probability distributions. Theoretical Computer Science, 282(1):101-150, 2002. A preliminary version of this paper appeared in Katoen [Kat99], pages 75-95.

[Koz81] Kozen, D.: Semantics of probabilistic programs. Journal of Computer and System Sciences, 22(3):328-350, 1981.

[KSK76] Kemeny, J. G., Snell, J. L. and Knapp, A. W: Denumerable Markov Chains, 2nd edition, Graduate Texts in Mathematics. Springer, 1976.

[LPY97] Larsen, K. G., Pettersson, P. and Yi, W.: UPPAAL in a nutshell. Software Tools for Technology Transfer, 1(1+2):134-152, 1997.

[LPY98] Lindahl, M., Pettersson, P. and Yi, W.: Formal design and analysis of a gear-box controller. In Steffen [Ste98], pages 281-297.

[Pri] PRISM: Web page: http: //www.cs.bham.ac.uk/ dxp/prism/

[Sad98] Sangiorgi, D. and de Simone, R., editors: Proceedings of the 9th International Conference on Concurrency Theory (CONCUR'98), volume 1466 of LNCS. Springer-Verlag, 1998.

[Seg95] Segala, R.: Modeling and Verification of Randomized Distributed Real-Time Systems. PhD thesis, MIT, Department of Electrical Engineering and Computer Science, 1995.

[SeL95] Segala, R. and Lynch, N. A.: Probabilistic simulations for probabilistic processes. Nordic Journal of Computing, 2(2):250-273, 1995.

[SiS01] Simons, D. P. L. and Stoelinga, M. I. A.: Mechanical verification of the IEEE 1394a root contention protocol using UPPAAL2k. Software Tools for Technology Transfer, 3(4):469-485, 2001.

[Ste98] Steffen, B., editor: Proceedings of the 4th Workshop on Tools and Algorithms for the Construction and Analysis of Systems (TACAS'98), volume 1384 of LNCS. Springer-Verlag, 1998.

[StV99] Stoelinga, M. I. A. and Vaandrager, F. W.: Root contention in IEEE 1394. In Katoen [Kat99], pages 53-74.

[Var85] Vardi, M. Y.: Automatic verification of probabilistic concurrent finite-state programs. In Proceedings of the 26th IEEE Symposium on Foundations of Computer Science (FOCS'85), pages 327-338. IEEE Computer Society Press, 1985.

[YPD94] Yi, W., Pettersson, P. and Daniels, M.: Automatic verification of real-time communicating systems by constraint-solving In D. Hogrefe and S. Leue, editors, Proceedings of the 7th International Conference on Formal Description Techniques (FORTE'94), pages 223-238. North-Holland, 1994.

Received July 2001

Accepted in revised form November 2002

\section{A. Proof of Theorem 2.5}

Before we can prove Theorem 2.5 we require the following definitions and lemmas. In the following, we suppose that the closed, diagonal-free probabilistic timed automaton PTA $=(L, \mathscr{X}, \Sigma, i n v$, prob $)$ is fixed. First, we introduce the relation $>\subseteq \mathbb{R}^{|\mathscr{X}|} \times \mathbb{N}^{|\mathscr{X}|}$ first presented in Beyer [Bey01], which associates with every real-valued clock valuation the set of its possible integer representatives. Recall that $\mathbf{k}_{x}$ denotes the greatest constant that the clock $x$ is compared to in the zones of PTA.

Definition A.1. For $v \in \mathbb{R}^{|\mathscr{X}|}$ and $v^{\prime} \in \mathbb{N}^{|\mathscr{X}|}, v>v^{\prime}$ holds if and only if there exists $t \in[0,1)$ such that for all $x \in \mathscr{X}$ one of the following conditions holds:

- $v^{\prime}(x)-1+t<v(x) \leqslant v^{\prime}(x)+t$

- $v^{\prime}(x)-1+t<v(x)$ and $v^{\prime}(x)=\mathbf{k}_{x}+1$.

The following lemma is immediate.

Lemma A.2. For any zone $\zeta$ of PTA and clock valuations $v \in \mathbb{R}^{|\mathscr{X}|}, v^{\prime} \in \mathbb{N}^{|\mathscr{X}|}$ such that $v>v^{\prime}$, if $v \triangleleft \zeta$ then $v^{\prime} \triangleleft \zeta$. 
In particular, the lemma means that, for any state $(l, v) \in S_{\mathbb{R}}$ of the continuous semantics $\llbracket P T A \rrbracket_{\mathbb{R}}$, then, for any $v^{\prime} \in \mathbb{N}^{|\mathscr{X}|}$ such that $v>v^{\prime}$, the pair $\left(l, v^{\prime}\right)$ is a state of the integer semantics $\llbracket \mathrm{PTA} \rrbracket_{\mathbb{N}}$ (that is, $\left(l, v^{\prime}\right) \in S_{\mathbb{N}}$ ), because $v \triangleleft \operatorname{inv}(l)$ implies $v^{\prime} \triangleleft \operatorname{inv}(l)$.

Next, for the probabilistic system $\llbracket \mathrm{PTA} \rrbracket_{\mathbb{T}}=(S$, Act , Steps $)$ and any adversary $C \in A d v_{\llbracket \mathrm{PTA}}$, we define a sequence of functions $\left(\mathbf{P}_{n}^{C}\right)_{n \in \mathbb{N}}$. Intuitively, for any finite path $\omega \in \operatorname{Path}_{\text {fin }}^{C}$ and set of locations $L^{\prime} \subseteq L, \mathbf{P}_{n}^{C}\left(\omega, L^{\prime}\right)$ equals the probability, according to the adversary $C$, of reaching a state in $F_{L^{\prime}}^{\mathbb{T}}$ in at most $n$ transitions after performing the path $\omega$. Formally we have the following definition.

Definition A.3. If $\llbracket P T A \rrbracket_{\mathbb{T}}=(S, A c t$, Steps $)$, then for any adversary $C \in A d v_{\mathbb{P P T A}_{\mathbb{T}}}, L^{\prime} \subseteq L$ and finite path $\omega \in \operatorname{Path}_{\text {fin }}^{C}$, let:

$$
\mathbf{P}_{0}^{C}\left(\omega, L^{\prime}\right)= \begin{cases}1 & \text { if last }(\omega) \in F_{L^{\prime}}^{\mathbb{T}} \\ 0 & \text { otherwise }\end{cases}
$$

and for any $n \in \mathbb{N}, \omega \in \operatorname{Path}_{\text {fin }}^{C}$, if $C(\omega)=(a, \mu)$ then:

$$
\mathbf{P}_{n+1}^{C}\left(\omega, L^{\prime}\right)=\left\{\begin{array}{cl}
1 & \text { if last }(\omega) \in F_{L^{\prime}}^{\mathbb{T}} \\
\sum_{s^{\prime} \in S} \mu\left(s^{\prime}\right) \cdot \mathbf{P}_{n}^{C}\left(\omega \stackrel{a, \mu}{\longrightarrow} s^{\prime}, L^{\prime}\right) & \text { otherwise }
\end{array}\right.
$$

We now give Lemma A.4, which follows from classical probabilistic reachability. We abuse notation by regarding a single state $s \in S$ as a path of length 0 (that is, a path without any transitions).

Lemma A.4. If $\llbracket P T A \rrbracket_{\mathbb{T}}=(S, A c t$, Steps $)$, then for any state $s \in S$ and $L^{\prime} \subseteq L$, we have:

$$
\begin{aligned}
& \text { MaxProbReach }_{\mathbb{P P T A} \mathbb{\mathbb { T }}_{\mathbb{T}}}\left(s, F_{L^{\prime}}^{\mathbb{T}}\right) \stackrel{\text { def }}{=} \sup _{C \in \operatorname{Adv}\left[\mathrm{PTA}_{\mathbb{T}}\right.} \lim _{n \rightarrow \infty} \mathbf{P}_{n}^{C}\left(s, L^{\prime}\right) \\
& \text { MinProbReach }_{\mathbb{P P T A}_{\mathbb{T}}}\left(s, F_{L^{\prime}}^{\mathbb{T}}\right) \stackrel{\text { def }}{=} \inf _{C \in \operatorname{Adv}_{[\mathrm{PTA}]_{\mathbb{T}}}} \lim _{n \rightarrow \infty} \mathbf{P}_{n}^{C}\left(s, L^{\prime}\right)
\end{aligned}
$$

Finally, we require the following lemma.

Lemma A.5. Let PTA $=(L, \mathscr{X}, \Sigma$, inv, prob $)$ be a closed, diagonal-free probabilistic timed automaton, with continuous semantics $\llbracket \mathrm{PTA} \rrbracket_{\mathbb{R}}=\left(S_{\mathbb{R}}, \mathbb{R} \cup \Sigma\right.$, Steps $\left.\mathbb{R}_{\mathbb{R}}\right)$ and integer semantics $\llbracket \mathrm{PTA} \rrbracket_{\mathbb{N}}=\left(S_{\mathbb{N}}, \mathbb{N} \cup \Sigma\right.$, Steps $\left.s_{\mathbb{N}}\right)$. For all $L^{\prime} \subseteq L, n \in \mathbb{N},(l, v) \in S_{\mathbb{R}}, A \in A d v_{\mathbb{P P A} \|_{\mathbb{R}}}$ and $v^{\prime} \in \mathbb{N}^{|\mathscr{X}|}$ such that $v>v^{\prime}$, there exist adversaries $B_{1}, B_{2} \in A d v_{\llbracket P T A \rrbracket_{N}}$ such that:

$$
\mathbf{P}_{n}^{B_{1}}\left(\left(l, v^{\prime}\right), L^{\prime}\right) \leqslant \mathbf{P}_{n}^{A}\left((l, v), L^{\prime}\right) \leqslant \mathbf{P}_{n}^{B_{2}}\left(\left(l, v^{\prime}\right), L^{\prime}\right)
$$

Proof. If $l \in L^{\prime}$ then, by Definition A.3, for any adversary $B \in A d v_{\llbracket P T A} \mathbb{N}_{\mathbb{N}}$ and $n \in \mathbb{N}$, we have $\mathbf{P}_{n}^{A}\left((l, v), L^{\prime}\right)=$ $\mathbf{P}_{n}^{B}\left(\left(l, v^{\prime}\right), L^{\prime}\right)=1$, and hence the lemma holds in this case.

We are left to consider the case when $l \notin L^{\prime}$. We proceed by induction on $n \in \mathbb{N}$. For $n=0$, the result follows from the fact that for any adversary $B \in A d v_{\llbracket P T A \rrbracket_{\mathrm{N}}}$ :

$$
\mathbf{P}_{0}^{A}\left((l, v), L^{\prime}\right)=\mathbf{P}_{0}^{B}\left(\left(l, v^{\prime}\right), L^{\prime}\right)=0
$$

Now suppose that the lemma holds for some $n \in \mathbb{N}$. By Definition 2.4, we have the following two cases to consider:

Time Transitions. $A(l, v)=(t, \mu)$ for some $t \in \mathbb{R}$ such that $v+\tilde{t} \triangleleft \operatorname{inv}(l)$ for all $0 \leqslant \tilde{t} \leqslant t$ and $\mu(l, v+t)=1$. In this case, for any $\left(l, v^{\prime}\right) \in S_{\mathbb{N}}$, if $v>v^{\prime}$, then from Lemma 1 of Beyer [Bey01] there exists $t^{\prime} \in \mathbb{N}$ such that:

$$
v^{\prime} \oplus_{\mathbb{N}} t^{\prime} \triangleleft \operatorname{inv}(l) \text { and } v+t>v^{\prime} \oplus_{\mathbb{N}} t^{\prime}
$$

Let $A^{\prime} \in A d v_{\mathbb{P P T}_{\mathbb{R}_{\mathbb{R}}}}$ be an adversary such that $A^{\prime}(\omega)=A((l, v) \stackrel{t, \mu}{\rightarrow} \omega)$. Then, by Definition A.3 and the fact that $\mu(l, v+t)=1$, we have $\mathbf{P}_{n+1}^{A}\left((l, v), L^{\prime}\right)=\mathbf{P}_{n}^{A^{\prime}}\left((l, v+t), L^{\prime}\right)$, and, by induction and (1), there exist $B_{1}^{\prime}, B_{2}^{\prime} \in$ $A d v_{\llbracket \mathrm{PTA} \rrbracket_{\mathrm{N}}}$ such that:

$$
\mathbf{P}_{n}^{B_{1}^{\prime}}\left(\left(l, v^{\prime} \oplus_{\mathbb{N}} t^{\prime}\right), L^{\prime}\right) \leqslant \mathbf{P}_{n}^{A^{\prime}}\left((l, v+t), L^{\prime}\right) \leqslant \mathbf{P}_{n}^{B_{2}^{\prime}}\left(\left(l, v^{\prime} \oplus_{\mathbb{N}} t^{\prime}\right), L^{\prime}\right)
$$


Furthermore, by (1) and Definition 2.4, there exists $\left((l, v), t^{\prime}, \mu^{\prime}\right) \in \operatorname{Steps}_{\mathbb{N}}$ with $\mu^{\prime}\left(l, v^{\prime} \oplus_{\mathbb{N}} t^{\prime}\right)=1$. Therefore, letting $B_{1}$ and $B_{2}$ be the adversaries which choose the transition $\left(t^{\prime}, \mu^{\prime}\right)$ in $\left(l, v^{\prime}\right)$ and then behave like $B_{1}^{\prime}$ and $B_{2}^{\prime}$ respectively, by Definition A.3 we have $\mathbf{P}_{n+1}^{B_{1}}\left((l, v), L^{\prime}\right)=\mathbf{P}_{n}^{B_{1}^{\prime}}\left(\left(l, v^{\prime} \oplus_{\mathbb{N}} t^{\prime}\right), L^{\prime}\right)$ and $\mathbf{P}_{n+1}^{B_{2}}\left(\left(l, v^{\prime}\right), L^{\prime}\right)=$ $\mathbf{P}_{n}^{B_{2}^{\prime}}\left(\left(l, v^{\prime} \oplus_{\mathbb{N}} t^{\prime}\right), L^{\prime}\right)$. The required result then follows from (2).

Discrete Transitions. $A(l, v)=(\sigma, \mu)$ for some $\sigma \in \Sigma$ such that there exists $(l, g, \sigma, p) \in$ prob with $v \triangleleft g$ and for all $\left(l^{\prime}, w\right) \in S_{\mathbb{R}}:$

$$
\mu\left(l^{\prime}, w\right)=\sum_{X \subseteq \mathscr{X} \& w=v[X:=0]} p\left(X, l^{\prime}\right)
$$

In this case, for any $\left(l, v^{\prime}\right) \in S_{\mathbb{N}}$ such that $v>v^{\prime}$, it follows from Lemma A.2 and the definition of $>$ that $v^{\prime} \triangleleft g$. Now by Definition 2.4 there exists $\left(\left(l, v^{\prime}\right), \sigma, \mu^{\prime}\right) \in \operatorname{Steps}_{\mathbb{N}}$ such that, for all $\left(l^{\prime}, w^{\prime}\right) \in S_{\mathbb{N}}$ :

$$
\mu^{\prime}\left(l^{\prime}, w^{\prime}\right)=\sum_{X \subseteq \mathscr{X} \& w^{\prime}=v^{\prime}[X:=0]} p\left(X, l^{\prime}\right)
$$

Consider any $\left(l^{\prime}, X\right) \in L \times 2^{\mathscr{X}}$ such that $p\left(l^{\prime}, X\right)>0$. Let $A^{l^{\prime}, v[X:=0]}$ be the adversary such that $A^{l^{\prime}, v[X:=0]}(\omega)=$ $A((l, v) \stackrel{\sigma, \mu}{\longrightarrow} \omega)$ for any finite path $\omega$ such that $\omega(0)=\left(l^{\prime}, v[X:=0]\right)$. Then by Definition A.3 we have:

$$
\begin{aligned}
\mathbf{P}_{n+1}^{A}\left((l, v), L^{\prime}\right) & =\sum_{\left(l^{\prime}, w\right) \in S_{\mathbb{R}}} \mu\left(l^{\prime}, w\right) \cdot \mathbf{P}_{n}^{A}\left((l, v) \stackrel{\sigma, \mu}{\longrightarrow}\left(l^{\prime}, w\right), L^{\prime}\right) & & \\
& =\sum_{\left(l^{\prime}, w\right) \in S_{\mathbb{R}}}\left(\sum_{X \subseteq \mathscr{X} \& w=v[X:=0]} p\left(X, l^{\prime}\right)\right) \cdot \mathbf{P}_{n}^{A}\left(\left(l, v^{\prime}\right) \stackrel{\sigma, \mu}{\longrightarrow}\left(l^{\prime}, w\right), L^{\prime}\right) & & \text { by (3) } \\
& =\sum_{l^{\prime} \in L \& X \subseteq \mathscr{X}} p\left(X, l^{\prime}\right) \cdot \mathbf{P}_{n}^{A}\left((l, v) \stackrel{\sigma, \mu^{\prime}}{\longrightarrow}\left(l^{\prime}, v[X:=0]\right), L^{\prime}\right) & & \text { rearranging } \\
& =\sum_{l^{\prime} \in L \& X \subseteq \mathscr{X}} p\left(X, l^{\prime}\right) \cdot \mathbf{P}_{n}^{A^{l^{\prime}, v[X:=0]}}\left(\left(l^{\prime}, v[X:=0]\right), L^{\prime}\right) & & \text { by construction }
\end{aligned}
$$

Now, from Lemma 1 of Beyer [Bey01], since $v>v^{\prime}$, for any $X \subseteq \mathscr{X}$ we have $v[X:=0]>v^{\prime}[X:=0]$, and hence by induction there exists $B_{1}^{l^{\prime}, v[X:=0]} \in A d v_{\llbracket \mathrm{PTA} \rrbracket_{\mathrm{N}}}$ such that:

$$
\mathbf{P}_{n}^{B_{1}^{l^{\prime}, v[X:=0]}}\left(\left(l^{\prime}, v^{\prime}[X:=0]\right), L^{\prime}\right) \leqslant \mathbf{P}_{n}^{A^{l^{\prime}, v[X:=0]}}\left(\left(l^{\prime}, v[X:=0]\right), L^{\prime}\right)
$$

For any $X \subseteq \mathscr{X}$, let $B_{1}^{l^{\prime}, v^{\prime}[X:=0]}$ be the adversary from the set

$$
\left\{B_{1}^{l^{\prime}, v\left[X^{\prime}:=0\right]} \mid X^{\prime} \subseteq \mathscr{X} \wedge v^{\prime}[X:=0]=v^{\prime}\left[X^{\prime}:=0\right]\right\}
$$

which minimises the value of $\mathbf{P}_{n}^{B_{1}^{l^{\prime}, v[X:=0]}}\left(\left(l^{\prime}, v^{\prime}[X:=0]\right), L^{\prime}\right)$. Finally, letting $B_{1}$ be the adversary that chooses the transition $\left(\sigma, \mu^{\prime}\right)$ in $\left(l, v^{\prime}\right)$ and then behaves like $B_{1}^{l^{\prime}, v^{\prime}[X:=0]}$ in the state $\left(l^{\prime}, v^{\prime}[X:=0]\right)$, using Definition A.3 and (4) it follows that:

$$
\begin{aligned}
& \mathbf{P}_{n+1}^{B_{1}}\left(\left(l, v^{\prime}\right), L^{\prime}\right)=\sum_{l^{\prime} \in L \& X \subseteq \mathscr{X}} p\left(X, l^{\prime}\right) \cdot \mathbf{P}_{n}^{B_{1}^{l^{\prime}, v^{\prime}[X:=0]}}\left(\left(l^{\prime}, v^{\prime}[X:=0]\right), L^{\prime}\right) \\
& \leqslant \sum_{l^{\prime} \in L \& X \subseteq \mathscr{X}}^{l^{\prime} \in L \& X \subseteq \mathscr{X}} p\left(X, l^{\prime}\right) \cdot \mathbf{P}_{n}^{B^{l^{\prime}, v[X:=0]}}\left(\left(l^{\prime}, v^{\prime}[X:=0]\right), L^{\prime}\right) \quad \text { by construction } \\
& \leqslant \sum_{l^{\prime} \in L \& X \subseteq \mathscr{X}}^{l^{\prime} \in L \& X \subseteq \mathscr{X}} p\left(X, l^{\prime}\right) \cdot \mathbf{P}_{n}^{A^{l^{\prime}, v[X:=0]}}\left(\left(l^{\prime}, v[X:=0]\right), L^{\prime}\right) \quad \text { by }(5) \\
& =\stackrel{P}{n+1}_{n}^{A}\left(\left(l^{\prime}, v[X:=0]\right), L^{\prime}\right) \quad \text { from above }
\end{aligned}
$$

Showing that $\mathbf{P}_{n+1}^{A}\left((l, v), L^{\prime}\right) \leqslant \mathbf{P}_{n+1}^{B_{2}}\left(\left(l, v^{\prime}\right), L^{\prime}\right)$ for some $B_{2} \in A d v_{\llbracket \mathrm{PTA} \rrbracket_{\mathbb{N}}}$ follows similarly. 
We now give the proof of Theorem 2.5, that is, for every closed, diagonal-free probabilistic timed automata PTA $=(L, \mathscr{X}, \Sigma$, inv, prob $)$, initial location $\bar{l} \in L$, and target locations $L^{\prime} \subseteq L$ :

$$
\begin{aligned}
& \operatorname{MaxProbReach}_{\mathbb{I P T A} \mathbb{\mathbb { R }}_{\mathbb{R}}}\left((\bar{l}, \mathbf{0}), F_{\mathbb{R}}^{L^{\prime}}\right)=\text { MaxProbReach }_{\mathbb{P P T A} \mathbb{N}_{\mathbb{N}}}\left((\bar{l}, \mathbf{0}), F_{\mathbb{N}}^{L^{\prime}}\right) \\
& \text { MinProbReach }_{\mathbb{P P T A} \mathbb{I}_{\mathbb{R}}}\left((\bar{l}, \mathbf{0}), F_{\mathbb{R}}^{L^{\prime}}\right)=\text { MinProbReach }_{\mathbb{P P T A} \mathbb{N}_{\mathbb{N}}}\left((\bar{l}, \mathbf{0}), F_{\mathbb{N}}^{L^{\prime}}\right)
\end{aligned}
$$

Proof of Theorem 2.5 Since $(\bar{l}, \mathbf{0})>(\bar{l}, \mathbf{0})$, by Lemma A.5 and Lemma A.4 if follows that:

$$
\begin{aligned}
& \text { MaxProbReach }_{\mathbb{I P T A} \mathbb{\mathbb { R }}_{\mathbb{R}}}\left((\bar{l}, \mathbf{0}), F_{\mathbb{R}}^{L^{\prime}}\right) \leqslant \text { MaxProbReach }_{\mathbb{P P T A} \mathbb{N}_{\mathbb{N}}}\left((\bar{l}, \mathbf{0}), F_{\mathbb{N}}^{L^{\prime}}\right) \\
& \text { MinProbReach }_{\mathbb{P P T A} \mathbb{\mathbb { R }}_{\mathbb{R}}}\left((\bar{l}, \mathbf{0}), F_{\mathbb{R}}^{L^{\prime}}\right) \geqslant \text { MinProbReach }_{\mathbb{P P T A}}\left((\bar{l}, \mathbf{0}), F_{\mathbb{N}}^{L^{\prime}}\right)
\end{aligned}
$$

On the other hand, by definition of the continuous and integer semantics of PTA for any adversary $B \in A d v_{\mathbb{P P T A} \rrbracket_{\mathbb{N}}}$ there exists an adversary $A \in A d v_{\mathbb{I P T A}_{\mathbb{R}}}$ such that:

$$
\mathbf{P}_{n}^{A}\left((\bar{l}, \mathbf{0}), L^{\prime}\right)=\mathbf{P}_{n}^{B}\left((\bar{l}, \mathbf{0}), L^{\prime}\right)
$$

for all $n \in \mathbb{N}$. Intuitively, the adversary $A$ behaves like an integer semantics adversary; that is, it chooses to make timed transitions of only integer duration. Since this was for an arbitrary $B \in A d v_{\llbracket \text { PTA }} \mathbb{N}_{\mathbb{N}}$, it follows that:

$$
\begin{aligned}
& \operatorname{MaxProbReach}_{\mathbb{I P T A} \mathbb{\mathbb { R }}_{\mathbb{R}}}\left((\bar{l}, \mathbf{0}), F_{\mathbb{R}}^{L^{\prime}}\right) \geqslant \text { MaxProbReach }_{\mathbb{P P T A} \mathbb{N}_{\mathbb{N}}}\left((\bar{l}, \mathbf{0}), F_{\mathbb{N}}^{L^{\prime}}\right) \\
& \text { MinProbReach }_{\mathbb{P P T A} \mathbb{\mathbb { R }}_{\mathbb{R}}}\left((\bar{l}, \mathbf{0}), F_{\mathbb{R}}^{L^{\prime}}\right) \leqslant \text { MinProbReach }_{\mathbb{P P T A} \mathbb{N}_{\mathbb{N}}}\left((\bar{l}, \mathbf{0}), F_{\mathbb{N}}^{L^{\prime}}\right)
\end{aligned}
$$

Putting the above together we have

$$
\begin{aligned}
& \operatorname{MaxProbReach}_{\mathbb{I P T A} \mathbb{\mathbb { R }}_{\mathbb{R}}}\left((\bar{l}, \mathbf{0}), F_{\mathbb{R}}^{L^{\prime}}\right)=\text { MaxProbReach }_{\mathbb{P P T A} \mathbb{N}_{\mathbb{N}}}\left((\bar{l}, \mathbf{0}), F_{\mathbb{N}}^{L^{\prime}}\right) \\
& \operatorname{MinProbReach}_{\mathbb{\mathbb { P T A }} \mathbb{\mathbb { R }}_{\mathbb{R}}}\left((\bar{l}, \mathbf{0}), F_{\mathbb{R}}^{L^{\prime}}\right)=\text { MinProbReach }_{\mathbb{P P T A}_{\mathbb{N}}}\left((\bar{l}, \mathbf{0}), F_{\mathbb{N}}^{L^{\prime}}\right)
\end{aligned}
$$

as required.

\section{B. Proof of Lemma 4.1}

Proof. Consider the path formula $\square \neg($ done $\wedge y \leqslant$ deadline). By the distributivity of conjunction, $\square \neg($ done $\wedge y \leqslant$ deadline $) \equiv \square((\neg$ done $) \vee y>$ deadline $)$. We now show that $\omega \models_{\mathscr{d}} \square((\neg$ done $) \vee y>$ deadline $)$ if and only if $\omega \models{ }_{\mathscr{A}} \diamond$ deadline_exceeded.

$(\Rightarrow)$ From $\omega \models g, \square((\neg$ done $) \vee y>$ deadline $)$, we have ( $\neg$ done) continuously for all states corresponding to times in the interval $[0$, deadline]. Consider the final transition made from a state at a time in the interval [0, deadline]. Firstly, note that this transition exists, because the path is non-Zeno and $y$ is never reset; secondly, observe that it must be a continuous transition, as discrete transitions are instantaneous. The location of both the source and target state of this transition must be other than done, because time transitions must have the same location in both their source and target state. From inspection of $\mathrm{I}_{1}^{\mathrm{p}+}$, we have $y \leqslant$ deadline in all locations apart from done and deadline_exceeded. Hence, the source and target location of the transition must be deadline_exceeded, and therefore $\diamond$ deadline_exceeded holds for this path.

$(\Leftarrow)$ From inspection of $\mathrm{I}_{1}^{\mathrm{p}+}$, we have $\omega=_{\mathcal{A}} \diamond$ deadline_exceeded implies $\omega=_{\triangleleft} \square(\neg$ done), as it is impossible to reach deadline_exceeded from done, and vice versa. Then clearly $\square(\neg$ done) implies $\square((\neg$ done $) \vee y>$ deadline $)$.

\section{Proof of Lemma 4.3}

Before giving the proof of Lemma 4.3, we present two auxiliary lemmas. The following lemma establishes that there does not exist a state of $\mathrm{I}_{1}^{\mathrm{p}+}$ from which it is impossible for some adversary to let time advance unboundedly with probability 1 .

Lemma C.1. For all states $s \in S$ of $\llbracket I_{1}^{\mathrm{p}+} \rrbracket_{\mathbb{R}}$, there exists an adversary $A$ of $\llbracket \mathrm{I}_{1}^{\mathrm{p}+} \rrbracket_{\mathbb{R}}$ such that:

$$
\operatorname{Prob}^{A}\left\{\omega \in \operatorname{Path}_{\text {ful }}^{A} \mid \omega(0)=s \& \omega \text { is non-Zeno }\right\}=1
$$


Proof. We proceed by contradiction: assume that there exists a state of $\llbracket \mathrm{I}_{1}^{\mathrm{p}+} \rrbracket_{\mathbb{R}}$ such that there does not exist a 'non-Zeno' adversary which meets the criteria given in the lemma. A necessary (but not sufficient) condition for the existence of such a state is that either (1) an invariant of a location imposes an upper bound on at least one clock which is greater than the upper bound on the same clock included in the enabling conditions for all of the location's outgoing edges, or (2) a loop in the graph derived from $\mathrm{I}_{1}^{\mathrm{p}+}$ features locations whose invariants impose an upper bound on at least one clock which is not reset within the loop. Neither of these conditions is satisfied in $\mathrm{I}_{1}^{\mathrm{p}+}$, and therefore a state from which there does not exist a 'non-Zeno' adversary is not possible.

We now examine the Zeno paths of the probabilistic timed automaton $\mathrm{I}_{1}^{\mathrm{p}+}$. The following lemma expresses the fact that the location of $\mathrm{I}_{1}^{\mathrm{p}+}$ cannot change infinitely often in a finite amount of time.

Lemma C.2. Let $\omega$ be a Zeno path of $\llbracket I_{1}^{p+} \rrbracket_{\mathbb{R}}$. Then there exists an infinite suffix $\omega^{\text {suf }}$ of $\omega$ such that all of its states feature the same location.

Proof. Each loop of the graph given by the locations and edges of $\mathrm{I}_{1}^{\mathrm{p}+}$ features (1) an enabling condition of the form $x \geqslant c$ for some $c>0$, and (2) a clock reset of $x$. Hence, if a path exhibits infinitely many edge traversals, then it must be non-Zeno.

For any finite path $\omega$ and infinite path $\omega^{\prime}$ such that last $(\omega)=\omega^{\prime}(0)$ (i.e. the last state of $\omega$ equals the first state of $\left.\omega^{\prime}\right)$, let $\omega \omega^{\prime}$ denote the infinite path obtained by concatenating $\omega$ and $\omega^{\prime}$. Moreover, for any infinite path $\omega$, let $\omega^{(i)}$ denote the $i$ th prefix of $\omega$. We now proceed to the proof of the main lemma.

Proof of Lemma 4.3. Consider the following adversary $A_{n Z}$ of $\left[\llbracket \mathrm{I}_{1}^{\mathrm{p}+} \rrbracket_{\mathbb{R}}\right.$, defined as behaving in the same manner as $A$ on all finite prefixes of non-Zeno paths, and as behaving in a non-Zeno manner after some sufficiently long finite prefix of any Zeno path. Formally, let $j \in \mathbb{N}$ be some index such that the location component after the $j$ th state of all Zeno paths of $A$ is constant. Such an index exists by Lemma C.2. We then define the adversary $A_{n Z}$ in the following manner:

- for any non-Zeno path $\omega$ of the adversary $A$, let $A_{n Z}\left(\omega^{(i)}\right)=A\left(\omega^{(i)}\right)$ for all $i \in \mathbb{N}$;

- for any Zeno path $\omega$ of $A$, let $\tilde{A}$ be an adversary of $\llbracket \mathrm{I}_{1}^{\mathrm{p}+} \rrbracket_{\mathbb{R}}$ such that:

$$
\operatorname{Prob}^{\tilde{A}}\left\{\tilde{\omega} \in \operatorname{Path}_{\text {ful }}^{\tilde{A}} \mid \tilde{\omega}(0)=\omega(j) \& \tilde{\omega} \text { is non-Zeno }\right\}=1
$$

Such an adversary exists by Lemma C.1. Then let $A_{n Z}\left(\omega^{(i)}\right)=A\left(\omega^{(i)}\right)$ for all $0 \leqslant i \leqslant j$, and let $A_{n Z}\left(\omega^{(j)} \tilde{\omega}\right)=$ $\tilde{A}(\tilde{\omega})$ for all finite paths $\tilde{\omega}$ of $\tilde{A}$ such that $\tilde{\omega}(0)=\omega(j)$.

It follows that $A_{n Z}$ is a non-Zeno adversary of $\llbracket \mathrm{I}_{1}^{\mathrm{p}+} \rrbracket_{\mathbb{R}}$.

The inequality of Lemma 4.3 holds for the following reasons. It suffices to consider the cones generated by all finite paths of $A$ of length $j$. We consider two cases, depending on whether a cone corresponds to possibly many non-Zeno paths or to a single Zeno path.

Case: Non-Zeno Paths. By the definition of $A_{n Z}$, the adversaries $A$ and $A_{n Z}$ agree along both the finite path generating the cone and along all of the non-Zeno paths included in the cone. Hence, the cone of $A$ and the corresponding cone of $A_{n Z}$ have the same probability of reaching deadline_exceeded.

Case: Zeno Paths. Recall that, by Lemma C.2, after the $j$ th state, the cone of $A$ comprising a single infinite, Zeno path remains within the same location. We consider the following sub-cases depending on the identity of this location:

Sub-case: done. As done is unreachable from deadline_exceeded, and vice versa, the probability assigned by the cones to reaching deadline_exceeded is 0 in both $A$ and $A_{n Z}$.

Sub-case: deadline_exceeded. The probability assigned by the cones to reaching deadline_exceeded is 1 in both $A$ and $A_{n Z}$.

Sub-case: Location $l \in L \backslash\{$ done,deadline_exceeded\}. The probability assigned to the property of reaching deadline_exceeded by the cone of $A_{n Z}$ is at least the probability assigned to the same property by the cone 
of $A$, for the following reason. The finite path of $A$ generates a cone comprising a single infinite path which remains in the current location, thus reaching deadline_exceeded with probability 0 . Hence, the probability of reaching deadline_exceeded assigned by the cone of $A_{n Z}$ is at least that for the cone of $A$ (in fact, the location invariants, combined with the property of non-Zenoness of the cone of $A_{n Z}$, will ensure progress through the location of $\mathrm{I}_{1}^{\mathrm{p}+}$, in turn implying that the cone will feature probabilistic branching, and finally also implying that the probability of reaching deadline_exceeded is strictly greater than 0 ). 